\title{
Kolmogorov type inequalities for the Marchaud fractional derivatives on the real line and the half-line
}

\author{
Vladislav F Babenko, Mariya S Churilova, Nataliia V Parfinovych and Dmytro S Skorokhodov*
}

\section{"Correspondence:}

dmitriy.skorokhodov@gmail.com Department of Mechanics and Mathematics, Dnepropetrovsk National University, pr. Gagarina, 72 , Dnepropetrovsk, 49010, Ukraine

\begin{abstract}
In this paper we establish some new Kolmogorov type inequalities for the Marchaud and Hadamard fractional derivatives of the functions defined on a real axis or semi-axis. Simultaneously we solve two related problems: the Stechkin problem on the best approximation of unbounded operators by bounded ones on a given class of elements and the problem of optimal recovery of an operator on elements from some class given with prescribed error.
\end{abstract}

Keywords: inequalities for derivatives; fractional derivatives; approximation of unbounded operators by bounded; optimal recovery of operators; ideal lattice

\section{Introduction}

Inequalities estimating the norm of an intermediate derivative of a function in terms of the norm of the function itself and the norm of its higher-order derivative (inequalities of Kolmogorov type) are important in many areas of mathematics and its applications. Due to the efforts of many mathematicians, nowadays, a great number of sharp Kolmogorov type inequalities are known (see, for instance, surveys [1-3] and monographs [4-6]). In many questions of analysis and its applications the study of fractional-order derivatives is also important (see, for instance, [7]). For some known results on the Kolmogorov type inequalities for derivatives of fractional order we refer the reader to [8-15], the book [16, Chapter 2] and references therein.

In this paper we shall obtain some new Kolmogorov type inequalities for fractional derivatives. Simultaneously, we consider two closely related problems: the Stechkin problem on approximation of unbounded operators by bounded ones on a given class of elements $Q$, and the problem of optimal recovery of unbounded operator on the class $Q$ under assumption that elements in $Q$ are given with known error (for more information see $[1,2]$ and $[4$, Section 7.1$])$.

\subsection{The Kolmogorov type inequalities}

Let $G$ be the real line $\mathbb{R}=(-\infty,+\infty)$ or half-line $\mathbb{R}_{+}=[0,+\infty)$. By $L_{p}(G), 1 \leq p \leq \infty$, we denote the space of measurable functions $f: G \rightarrow \mathbb{R}$ whose modulus to the $p$ th power is

@2014 Babenko et al.; licensee Springer. This is an Open Access article distributed under the terms of the Creative Commons Attribution License (http://creativecommons.org/licenses/by/2.0), which permits unrestricted use, distribution, and reproduction in any medium, provided the original work is properly cited. 
integrable on $G$ (essentially bounded on $G$ if $p=\infty$ ), endowed with the standard norm

$$
\|f\|_{L_{p}(G)}:= \begin{cases}\left(\int_{G}|f(t)|^{p} d t\right)^{1 / p}, & \text { if } 1 \leq p<\infty \\ \operatorname{ess} \sup \{|f(t)|: t \in G\}, & \text { if } p=\infty\end{cases}
$$

For $r \in \mathbb{N}$ and $1 \leq s \leq \infty$, by $L_{p, s}^{r}(G)$ we denote the space of the functions $f \in L_{p}(G)$ having locally absolutely continuous on $G$ derivative $f^{(r-1)}$ and such that $f^{(r)} \in L_{s}(G)$.

Let $1 \leq q \leq \infty, k \in \mathbb{N} \cup\{0\}, 0 \leq k \leq r-1$, and $\lambda, \mu \in \mathbb{R}$. Inequalities of the form

$$
\left\|f^{(k)}\right\|_{L_{q}(G)} \leq K\|f\|_{L_{p}(G)}^{\mu}\left\|f^{(r)}\right\|_{L_{s}(G)}^{\lambda}
$$

holding true, for every function $f \in L_{p, s}^{r}(G)$ with some constant $K$ independent of $f$, are called Kolmogorov type inequalities (Kolmogorov-Nagy type inequalities when $k=0$ ). It is well known (see [17]) that the constant $K$ in inequality (1) is finite if and only if

$$
\lambda=\frac{k-1 / q+1 / p}{r-1 / s+1 / p}, \quad \mu=1-\lambda,
$$

and

$$
\frac{r}{q} \leq \frac{r-k}{p}+\frac{k}{s}
$$

Naturally, inequalities with the lowest possible (sharp) constant $K$ are of the most interest. We refer the reader to $[1,2]$ and the books $[4,6]$ for the detailed survey on the Kolmogorov type inequalities and discussion of related questions.

Together with inequalities (1) the study of inequalities between the norms of intermediate function derivative, the function itself and its higher-order derivative in spaces more general than $L_{p}$ are also important. In Sections 2-4 we shall obtain several inequalities between the norms of derivatives in ideal lattices (see [18, Chapter 2, Section 2]).

In this paper we focus on the study of the Kolmogorov type inequalities for non-integer (fractional) values of $k$. There are many ways to give a sense to the fractional derivative of a function defined on $\mathbb{R}$ or $\mathbb{R}_{+}$. Among the first ones was the fractional derivative in the Riemann-Liouville sense (see [7, Section 5.1]) that is defined for a function $f: \mathbb{R} \rightarrow \mathbb{R}$ and $x \in \mathbb{R}$, as follows:

$$
\mathcal{D}_{ \pm}^{k} f(x):=\frac{( \pm 1)^{n}}{\Gamma(n-k)} \cdot \frac{d^{n}}{d x^{n}} \int_{0}^{+\infty} t^{n-k-1} f(x \mp t) d t, \quad n=[k]+1,
$$

where $\Gamma(z)$ is the Euler gamma function and $[z]$ stands for the integer part of real number $z$. We shall mostly consider fractional derivatives in the Marchaud sense (see [19] or [7, Section 5.6]) that are defined for a function $f: \mathbb{R} \rightarrow \mathbb{R}$ and $x \in \mathbb{R}$, as follows:

$$
D_{ \pm}^{k} f(x)=\frac{1}{\varkappa(k, n)} \int_{0}^{+\infty} \frac{\left(\Delta_{ \pm t}^{n} f\right)(x)}{t^{1+k}} d t
$$


where $n \in \mathbb{N}, n>k$ (the definition itself is independent of $n$ ), and

$$
\begin{aligned}
& \left(\Delta_{ \pm}^{n} f\right)(x):=\sum_{m=0}^{n}(-1)^{m}\left(\begin{array}{c}
n \\
m
\end{array}\right) f(x \mp m t), \\
& \varkappa(k, n):=\Gamma(-k) \sum_{m=0}^{n}(-1)^{m}\left(\begin{array}{c}
n \\
m
\end{array}\right) m^{k} .
\end{aligned}
$$

For a function $f: \mathbb{R}_{+} \rightarrow \mathbb{R}$, the right hand sided derivatives $\mathcal{D}_{-}^{k} f$ and $D_{-}^{k} f$ are defined by formulas (4) and (5), respectively. The left hand sided derivatives $\mathcal{D}_{+}^{k} f$ and $D_{+}^{k} f$ are defined with the help of slightly different constructions (see [7, Sections 5.1, 5.5]), and we shall not study these derivatives here.

It is well known (see [7]) that $\mathcal{D}_{ \pm}^{k} f=D_{ \pm}^{k} f$ for 'good' functions $f: G \rightarrow \mathbb{R}$. However, construction (5) is also suitable for a wider class of the functions, e.g. constant functions or functions whose power growth at infinity has order lower than $k$.

Let us consider Kolmogorov type inequalities of the form (1) with the term $\left\|f^{(k)}\right\|_{L_{q}(G)}$ being replaced by $\left\|D_{-}^{k} f\right\|_{L_{q}(G)}$ in the left hand side:

$$
\left\|D_{-}^{k} f\right\|_{L_{q}(G)} \leq K\|f\|_{L_{p}(G)}^{\mu}\left\|f^{(r)}\right\|_{L_{s}(G)}^{\lambda}, \quad f \in L_{p, s}^{r}(G) .
$$

Similarly to inequalities (1) for derivatives of integer order, it is easy to see that the constant $K$ in (7) is finite only if parameters $\lambda$ and $\mu$ satisfy equalities (2).

Together with the Riemann-Liouville and the Marchaud fractional derivatives, Kolmogorov type inequalities were also studied $[15,20,21]$ for other fractional derivatives, e.g. the Riesz fractional derivative, the Weyl fractional derivative, etc. To the best of our knowledge, the sharp constant in inequality (7) was found in the following situations:

1. $G=\mathbb{R}, p=q=s=\infty, r=2$, and $k \in(0,1)$, - Geusberg [8];

2. $G=\mathbb{R}_{+}, p=q=s=\infty, r=2$, and $k \in(0,2) \backslash\{1\}$, - Arestov [10] (for $\mathcal{D}_{ \pm}^{k}$ );

3. $G=\mathbb{R}_{+}, p=q=s=\infty, 0<k \leq 1$, and $k<r \leq 2$, - Arestov [10] (for $\mathcal{D}_{ \pm}^{k}$ );

4. $G=\mathbb{R}$ or $G=\mathbb{R}_{+}, p=s=2, q=\infty, k<r$, - Buslaev and Tihomirov [21] (for the Weyl derivative);

5. $G=\mathbb{R}$ or $G=\mathbb{R}_{+}, p=q=\infty, 1 \leq s \leq \infty, r=1, k \in(0,1-1 / s)$, - Babenko and Churilova [22];

6. $G=\mathbb{R}, p=q=\infty, 1 \leq s \leq \infty, r=1,2, k \in(0, r-1 / s) \backslash\{1\}$, - Babenko and Parfinovych [20] and Babenko, Parfinovych and Pichugov [15] (for the Riesz derivative).

Here we establish sufficient conditions which allow writing sharp Kolmogorov type inequalities. Specifically, we focus on inequalities between the uniform norms of the function and its derivatives; the uniform norms of the function and its intermediate derivative and the norm of its higher-order derivative in the ideal lattice; the norms of the function and its derivatives in the ideal lattice. As a consequence in Section 4 we obtain several new sharp Kolmogorov type inequalities in the following cases:

1. $G=\mathbb{R}$ or $G=\mathbb{R}_{+}, p=q=\infty, r=1$, and $k \in(0,1)$, the norm of $f^{\prime}$ is considered in an ideal lattice;

2. $G=\mathbb{R}, p=q=s=1, r=1$, and $k \in(0,1)$;

3. $G=\mathbb{R}_{+}, p=q=\infty, r=2$, and $k \in(0,1)$, the norm of $f^{\prime \prime}$ is considered in an ideal lattice; 
4. $G=\mathbb{R}, p=q=\infty, 1 \leq s \leq \infty, r=2$, and $k \in(0,1)$;

5. $G=\mathbb{R}$ or $G=\mathbb{R}_{+}, p=q=\infty, 1 \leq s \leq \infty, r=2$, and $k \in(1,2-1 / s)$.

\subsection{The Stechkin problem}

The problem of the best approximation of unbounded operators by linear bounded ones is close to the problem of finding sharp constants in inequalities (1), (7) and, furthermore, presents an independent interest. We follow [23] (see also surveys [1, 2]) to set the problem rigorously.

Let $X$ and $Y$ be the Banach spaces; $A: X \rightarrow Y$ be an operator (not necessarily linear) with domain of definition $D_{A} \subset X ; Q \subset D_{A}$ be some set. The function

$$
\Omega(\delta)=\Omega(\delta, A, Q):=\sup \left\{\|A f\|_{Y}: f \in Q,\|f\|_{X} \leq \delta\right\}, \quad \delta \geq 0,
$$

is called the modulus of continuity of the operator $A$ on the set $Q$.

By $\mathcal{L}=\mathcal{L}(X, Y)$ we denote the space of all linear bounded operators $S: X \rightarrow Y$. The error of approximation of the operator $A$ by linear bounded operator $S \in \mathcal{L}$ on the set $Q$ is defined by

$$
U(A, S ; Q):=\sup _{x \in Q}\|A x-S x\|_{Y} .
$$

For $N>0$, we set

$$
E_{N}(A ; Q):=\inf _{S \in \mathcal{L},\|S\| \leq N} U(A, S ; Q)
$$

The Stechkin problem on the best approximation of the operator $A$ by linear bounded operators on set $Q$ consists in evaluating quantity (9) and finding extremal operators (if any exists) delivering an infimum in the right hand part of (9).

Now, we let

$$
\ell(\delta, A ; Q):=\inf _{N \geq 0}\left(E_{N}(A ; Q)+N \delta\right) .
$$

The following theorem by Stechkin [23] (see also [2, 4]) provides a simple but nevertheless effective lower estimate of quantity (9) in terms of the modulus of continuity $\Omega$.

Theorem A If $A$ is a homogeneous (in particular, linear) operator, $Q \subset D_{A}$ is centrally symmetric convex set, then, for every $N \geq 0$ and $\delta \geq 0$,

$$
\begin{aligned}
& E_{N}(A ; Q) \geq \sup _{\delta \geq 0}\{\Omega(\delta, A ; Q)-N \delta\}=\sup _{x \in Q}\left\{\|A x\|_{Y}-N\|x\|_{X}\right\}, \\
& \Omega(\delta, A ; Q) \leq \ell(\delta, A ; Q) .
\end{aligned}
$$

Furthermore, if there exists a pair of element $x_{0} \in Q$ and operator $S_{0} \in \mathcal{L}$ such that

$$
\left\|A x_{0}\right\|_{Y}=U\left(A, S_{0} ; Q\right)+\left\|S_{0}\right\| \cdot\left\|x_{0}\right\|_{X}
$$


then $\Omega\left(\left\|x_{0}\right\|_{X}, A ; Q\right)=\left\|A x_{0}\right\|_{Y}$ and

$$
E_{\left\|S_{0}\right\|}(A ; Q)=U\left(A, S_{0} ; Q\right)=\left\|A x_{0}\right\|_{Y}-\left\|S_{0}\right\| \cdot\left\|x_{0}\right\|_{X} .
$$

Consequently, the operator $S_{0}$ is extremal in problem (9) for $N=\left\|S_{0}\right\|$, and the element $x_{0}$ in problem (8) for $\delta=\left\|x_{0}\right\|_{X}$.

We refer the reader to [1,2] for a survey of other known results on the Stechkin problem and a discussion of related questions.

In the particular case when $G=\mathbb{R}$ or $G=\mathbb{R}_{+}, X=L_{p}(G), Y=L_{\infty}(G), A=D_{-}^{k}$, and

$$
Q=W_{p, s}^{r}(G):=\left\{f \in L_{p, s}^{r}(G):\left\|f^{(r)}\right\|_{L_{s}(G)} \leq 1\right\}
$$

for every $\delta \geq 0$, we have

$$
\Omega\left(\delta, D_{-}^{k} ; W_{p, s}^{r}(G)\right)=K \cdot \delta^{1-\lambda}, \quad \lambda=\frac{k+1 / p}{r-1 / s+1 / p},
$$

where $K=\Omega\left(1, D_{-}^{k} ; W_{p, s}^{r}(G)\right)$ is the sharp constant in inequality (7) with $q=\infty$. From the result of Gabushin [24, Lemma 1] it can easily be shown that estimate (10) is sharp when $Y=L_{\infty}(G)$. Hence, for every $N \geq 0$,

$$
E_{N}\left(D_{-}^{k} ; W_{p, s}^{r}(G)\right)=\sup _{\delta \geq 0}\left\{K \delta^{1-\lambda}-N \delta\right\}=\lambda(1-\lambda)^{\frac{1}{\lambda}-1} K^{\frac{1}{\lambda}} N^{1-\frac{1}{\lambda}} .
$$

Therefore, in all cases when the sharp constant $K$ in inequality (7) for $q=\infty$ is found, we immediately know the exact value of the quantity of the best approximation of the operator $D_{-}^{k}$ by linear bounded operators on the class $W_{p, s}^{r}(G)$.

\subsection{The problem of optimal recovery of operators on elements given with an error}

Another problem that is closely related to the Stechkin problem and sharp Kolmogorov type inequalities is the problem of optimal recovery of an operator with the help of the set of linear operators (or mappings in general) on elements of some set that are given with an error. We follow [2] to set the problem rigorously.

Let $X$ and $Y$ be the Banach spaces; $A: X \rightarrow Y$ be an operator (not necessarily linear) with domain of definition $D_{A} \subset Q ; Q \subset D_{A}$ be some set. By $\mathscr{R}$ we denote either the set $\mathscr{L}$ of all linear operators acting from $X$ to $Y$, or the set of all mappings $\mathscr{O}$ from $X$ to $Y$. For an arbitrary $\delta \geq 0$ and $S \in \mathscr{R}$, we set

$$
U_{\delta}(A, S ; Q):=\sup \left\{\|A f-S f\|_{Y}: f \in Q, g \in X,\|f-g\|_{X} \leq \delta\right\} .
$$

It is clear that $U_{0}(A, S ; Q)=U(A, S ; Q)$. The problem of optimal recovery of the operator $A$ with the help of the set of operators $\mathscr{R}$ on elements of the set $Q$ with given error $\delta$ consists in finding the quantity

$$
\mathcal{E}_{\delta}(\mathscr{R}, A ; Q):=\inf _{S \in \mathscr{R}} U_{\delta}(A, S ; Q)
$$


called the best recovery of the operator $A$ with the help of mappings from $\mathscr{R}$ on elements $Q$ given with prescribed error $\delta$. The detailed survey of existing results and further references can be found, for instance, in [2]. The following statement is a corollary of the result by Arestov [2, Theorem 2.1] that indicates the close relations between this problem and the Stechkin problem.

Theorem B If $A$ is a homogeneous (in particular, linear) operator, $Q \subset D_{A}$ is a centrally symmetric convex set, then, for every $N \geq 0$ and $\delta \geq 0$,

$$
\Omega(\delta, A ; Q) \leq \mathcal{E}_{\delta}(\mathscr{O}, A ; Q) \leq \mathcal{E}_{\delta}(\mathscr{L}, A ; Q) \leq \ell(\delta, A ; Q) .
$$

Moreover, if there exist an element $x_{0} \in Q$ and an operator $S_{0} \in \mathcal{L}(X, Y)$ satisfying (11) from Theorem A then for $\delta=\left\|x_{0}\right\|_{X}$,

$$
\left\|A x_{0}\right\|_{Y}=\Omega(\delta, A ; Q)=\mathcal{E}_{\delta}(\mathscr{O}, A ; Q)=\mathcal{E}_{\delta}(\mathscr{L}, A ; Q) .
$$

Similarly to the Stechkin problem, in the case $G=\mathbb{R}$ or $G=\mathbb{R}_{+}, X=L_{p}(G)$, and $Y=$ $L_{\infty}(G)$, for every $\delta>0$, we have

$$
\mathcal{E}_{\delta}\left(\mathscr{O}, D_{-}^{k} ; W_{p, s}^{r}(G)\right)=\mathcal{E}_{\delta}\left(\mathscr{L}, D_{-}^{k} ; W_{p, s}^{r}(G)\right)=\Omega\left(\delta, D_{-}^{k} ; W_{p, s}^{r}(G)\right)
$$

So once the sharp constant in inequality (7) is found, we immediately know the value of the error of optimal recovery of the operator $D_{-}^{k}$ by operators from $\mathscr{O}$ (or $\mathscr{L}$ ) on elements of the class $W_{p, s}^{r}(G)$ given with error $\delta$.

\subsection{Organization of the paper}

The paper is organized in the following way. Section 2 is devoted to auxiliary results concerning properties of the Marchaud fractional derivatives: existence, continuity, and integral representation in terms of the higher-order function derivative. Then we establish some sufficient conditions when sharp Kolmogorov type inequalities (7) can be written and derive some consequences from these conditions for $r=1,2$ in Section 4. Finally, in Section 5 we present applications of the main results: the Kolmogorov problem for three numbers consisting in finding necessary and sufficient conditions on the triple of real positive numbers that guarantee the existence of a function attaining these numbers as the norms of its three consecutive derivatives, and sharp Kolmogorov type inequalities for the weighted norms of the Hadamard fractional derivatives.

\section{Auxiliary results}

In this section we formulate auxiliary propositions on the existence and continuity of the Marchaud fractional derivative and its integral representation in terms of the higher-order derivative. These and similar questions were studied by many mathematicians. For an overview of known results we refer the reader to the books $[7,25]$ and references therein.

\subsection{Definitions and results}

Let $G=\mathbb{R}$ or $G=\mathbb{R}_{+}$. By $\mathfrak{S}(G)$ we denote the space of measurable functions $f: G \rightarrow \mathbb{R}$. The linear space $E \subset \mathfrak{S}(G)$ endowed with the norm $\|\cdot\|_{E}$ is called the ideal lattice on $G$ 
(see [18, Chapter 2, Section 2]) if, for every $f \in E$ and $g \in \mathfrak{S}(G)$ such that $|g(x)| \leq|f(x)|$ a.e. on $G$, it follows that $g \in E$ and $\|g\|_{E} \leq\|f\|_{E}$. The set $A(E) \subset G$ is called the support of the ideal lattice $E$ if $f(x)=0$ for every $f \in E$ and $x \notin A(E)$. By $E^{1}$ we denote the associated space to $E$ (see [18, Chapter 2, Section 3]), i.e. the space of the functions $g \in \mathfrak{S}(G)$ such that supp $g \subset A(E)$ and

$$
\|g\|_{E^{1}}:=\sup _{\substack{f \in E,\|f\|_{E} \leq 1}} \int_{G} f(x) g(x) d x<\infty .
$$

It is clear that $E^{1}$ is the ideal lattice on $G$ and is a subspace in the space dual to $E$. Ideal lattices generalize many important spaces e.g. spaces $L_{p}(G), 1 \leq p \leq \infty$, the Orlicz spaces [26], the Lorentz spaces [18], the Marcinkiewicz spaces [18], etc.

In what follows we would also say that an ideal lattice $E$ is semi shift invariant if, for every $f \in E$ and $x \in G$, we have $f(\cdot+x) \in E$ and either $\|f(\cdot+x)\|_{E}=\|f\|_{E}$ if $G=\mathbb{R}$ or $\|f(\cdot+x)\|_{E} \leq$ $\|f\|_{E}$ if $G=\mathbb{R}_{+}$.

Let $r \in \mathbb{N}, k \in(0, r) \backslash \mathbb{N}$, and $F$ let be an ideal lattice. By $L_{\infty, E}^{r}(G)$ and $L_{F, E}^{r}(G)$ we denote the spaces of the functions $f \in L_{\infty}(G)$ and $f \in F$, respectively, such that $f^{(r-1)}$ is locally absolutely continuous on $G$ and $f^{(r)} \in E$. In addition, let $\chi_{B}$ stand for the characteristic (indicator) function of a measurable set $B \subset \mathbb{R}$.

Proposition 1 Let $G=\mathbb{R}$ or $G=\mathbb{R}_{+}, r \in \mathbb{N}, k \in(0, r) \backslash \mathbb{N}, E$ be a semi shift-invariant lattice on $G$ such that

$$
(\cdot)^{r-k-1} \chi_{(0,1)}(\cdot) \in E^{1}
$$

and

$$
\lim _{h \rightarrow 0^{+}}\left\|(\cdot)^{r-1-k} \chi_{(0, h)}(\cdot)\right\|_{E^{1}}=0,
$$

where $E^{1}$ is the associated space to $E$. Then $D_{-}^{k} f$ exists and is continuous on $G$, for every function $f \in L_{\infty, E}^{r}(G)$.

Proposition 2 Let $G=\mathbb{R}$ or $G=\mathbb{R}_{+}, r \in \mathbb{N}, k \in(0, r) \backslash \mathbb{N}$, E be a semi shift-invariant lattice on $G$ satisfying condition (12). Then, for every $f \in L_{\infty, E}^{r}(G)$,

$$
D_{-}^{k} f(\cdot)=\frac{(-1)^{r}}{\Gamma(r-k)} \int_{0}^{+\infty} t^{r-1-k} f^{(r)}(\cdot+t) d u
$$

Proposition 3 Let $G=\mathbb{R}$ or $G=\mathbb{R}_{+}, r \in \mathbb{N}, k \in(0, r) \backslash \mathbb{N}, E$ be a semi-shift invariant lattice on $G$ satisfying condition (12) and $F$ be a semi-shift invariant lattice on $G$ such that $\chi_{(0,1)} \in$ $F^{1}$. Then $D_{-}^{k} f(x)$ exists, for every $f \in L_{F, E}^{r}$ and $x \in G$, and the integral representation (14) for $D_{-}^{k} f$ holds true.

In particular, when $E=L_{s}(G), 1 \leq s \leq \infty$, both conditions (12) and (13) are equivalent to the inequality $k<r-1 / s$. So the following corollaries hold true.

Proposition 4 Let $G=\mathbb{R}$ or $G=\mathbb{R}_{+}, r \in \mathbb{N}, 1 \leq p, s \leq \infty$, and $k \in(0, r-1 / s) \backslash \mathbb{N}$. Then, for every $f \in L_{p, s}^{r}(G), D_{-}^{k} f$ exists and is continuous on $G$, and (14) holds true. 
Proposition 5 Let $G=\mathbb{R}$ or $G=\mathbb{R}_{+}, r \in \mathbb{N}, 1 \leq s \leq \infty$, and $k \in(0, r) \backslash \mathbb{N}$. Then $D_{-}^{k} f(x)$ exists, for every $f \in L_{s, s}^{r}(G)$ and $x \in G$, and (14) holds true.

\subsection{The proofs of auxiliary results}

For the sake of completeness, we prove Propositions 1-3. Preliminarily, we recall the definition of the $B$-splines and some of their properties (see, e.g., [27, Section 4.2]). The firstorder $B$-spline $N_{1}$ is the function $\chi_{(0,1)}$. For $r \geq 2$, the $r$-order $B$-spline $N_{r}$ is defined by

$$
N_{r}(x)=\int_{\mathbb{R}} N_{r-1}(x-t) N_{1}(t) d t=\int_{0}^{1} N_{r-1}(x-t) d t, \quad x \in \mathbb{R} .
$$

It is well known that $N_{r}$ is continuous and positive on $(0, r)$ function, compactly supported on $[0, r]$. Moreover (see Theorem 4.3 in [27]), for every $r$-times differentiable function $f: G \rightarrow \mathbb{R}$ and every $t>0$,

$$
\left(\Delta_{-t}^{r} f\right)(x)=(-1)^{r} t^{r} \int_{0}^{r} N_{r}(u) f^{(r)}(x+u t) d u, \quad x \in G .
$$

Proof of Proposition 1 Let a function $f \in L_{\infty, E}^{r}(G)$ and a point $x \in G$ be arbitrary. We observe that $\left|\left(\Delta_{-t}^{r} f\right)(x)\right| \leq 2^{r} \mid f \|_{L_{\infty}(G)}$, for every $t>0$. Hence, by definition (5), for every $h>0$, we have

$$
\begin{aligned}
\varkappa(k, r)\left|D_{-}^{k} f(x)\right| & =\left|\int_{0}^{+\infty} \frac{\left(\Delta_{-t}^{r} f\right)(x)}{t^{1+k}} d t\right| \\
& \leq\left|\int_{0}^{h} \frac{\left(\Delta_{-t}^{r} f\right)(x)}{t^{1+k}} d t\right|+\left|\int_{h}^{+\infty} \frac{\left(\Delta_{-t}^{r} f\right)(x)}{t^{1+k}} d t\right| \\
& \leq\left|\int_{0}^{h} \frac{\left(\Delta_{-t}^{r} f\right)(x)}{t^{1+k}} d t\right|+\frac{2^{r} \mid f \|_{L_{\infty}(G)}}{k h^{k}} .
\end{aligned}
$$

Using (15), changing variables, altering the order of integration and applying the Hölder inequality we obtain

$$
\begin{aligned}
\left|\int_{0}^{h} \frac{\left(\Delta_{-t}^{r} f\right)(x)}{t^{1+k}} d t\right| & =\left|\int_{0}^{h} \int_{0}^{r} \frac{N_{r}(u) f^{(r)}(x+u t)}{t^{k+1-r}} d u d t\right| \\
& =\left|\int_{0}^{h} \int_{0}^{r t} \frac{N_{r}(v / t) f^{(r)}(x+v)}{t^{k+2-r}} d v d t\right| \\
& =\left|\int_{0}^{r h} f^{(r)}(x+v) \int_{v / r}^{h} \frac{N_{r}(v / t)}{t^{k+2-r}} d t d v\right| \\
& \leq\left\|f^{(r)}\right\|_{E} \cdot\left\|\chi_{(0, r h)}(\cdot) \int_{(\cdot) / r}^{h} \frac{N_{r}((\cdot) / t)}{t^{k+2-r}} d t\right\|_{E^{1}} .
\end{aligned}
$$

It is easy to show that $N_{r}(x) \leq x^{r-1}$, for every $x \in[0, r]$. Hence, for every $v \in(0, r h)$,

$$
\int_{v / r}^{h} \frac{N_{r}(v / t)}{t^{k+2-r}} d t \leq v^{r-1} \int_{v / r}^{h} \frac{d t}{t^{k+1}} \leq \frac{v^{r-k-1}}{k r^{k}} .
$$

From the latter and estimate (16) we conclude that

$$
\varkappa(k, r)\left|D_{-}^{k} f(x)\right| \leq \frac{2^{r}\|f\|_{L_{\infty}(G)}}{k h^{k}}+\frac{\left\|(\cdot)^{r-1-k} \chi_{(0, r h)}(\cdot)\right\|_{E^{1}} \cdot\left\|f^{(r)}\right\|_{E}}{k r^{k}},
$$


which proves the existence and uniform boundedness of derivative $D_{-}^{k} f(x)$ at an arbitrary point $x \in G$.

Now, we turn to the proof of continuity of $D_{-}^{k} f$ on $G$. Let $x \in G$ be an arbitrary point. For every $\varepsilon>0$, there exist numbers $h>0$ and $H>0$ such that

$$
\frac{\left\|(\cdot)^{r-k-1} \chi_{(0, r h)}(\cdot)\right\|_{E^{1}} \cdot\left\|f^{(r)}\right\|_{E}}{k r^{k} \varkappa(k, r)}<\frac{\varepsilon}{6} \quad \text { and } \quad \frac{2^{r+1}\|f\|_{L_{\infty}(G)}}{k H^{k} \varkappa(k, r)}<\frac{\varepsilon}{3} .
$$

The function $f$ is continuous on $G$ and is uniformly continuous on $[h / 2, r H+r h / 2]$. Hence, there exists $\delta \in(0, h / 2)$ such that, for every $y^{\prime}, y^{\prime \prime} \in[h / 2, r H+r h / 2],\left|y^{\prime}-y^{\prime \prime}\right|<\delta$, we have $\left|f\left(y^{\prime}\right)-f\left(y^{\prime \prime}\right)\right|<2^{-r} k h^{k}|\varkappa(k, r)| \varepsilon$. Then using similar arguments to the proof of the existence of $D^{k} f$, we see that, for every $x, y \in G,|x-y|<\delta$, for the function $g(t)=f(x+t)-f(y+t)$ we have

$$
\begin{aligned}
\left|D_{-}^{k} f(x)-D_{-}^{k} f(y)\right| & \\
\leq & \frac{1}{\varkappa(k, r)}\left|\int_{0}^{+\infty} \frac{\left(\Delta_{-t}^{r} g\right)(0)}{t^{1+k}} d t\right| \\
\leq & \frac{1}{\varkappa(k, r)}\left(\left|\int_{0}^{h} \frac{\left(\Delta_{-t}^{r} g\right)(0)}{t^{1+k}} d t\right|+\left|\int_{h}^{H} \frac{\left(\Delta_{-t}^{r} g\right)(0)}{t^{1+k}} d t\right|+\left|\int_{H}^{+\infty} \frac{\left(\Delta_{-t}^{r} g\right)(0)}{t^{1+k}} d t\right|\right) \\
\leq & \frac{\left\|g^{(r)}\right\|_{E} \cdot \|(\cdot)^{r-k-1} \chi(0, r h)}{k r^{k} \varkappa(\cdot) \|_{E^{1}}}+\frac{2^{r}\|g\|_{L_{\infty}([h, r H])}}{k h^{k} \varkappa(k, r)}+\frac{2^{r}\|g\|_{L_{\infty}(G)}}{k H^{k} \varkappa(k, r)} \\
\leq & \frac{2\left\|f^{(r)}\right\|_{E} \cdot \|(\cdot)^{r-k-1} \chi(0, r h)}{k \cdot) \|_{E^{1}}}+\frac{2^{r}\|f(x+\cdot)-f(y+\cdot)\|_{L_{\infty}([, r H])}}{k h^{k} \varkappa(k, r)} \\
& +\frac{2^{r+1}\|f\|_{L_{\infty}(G)}}{k H^{k} \varkappa(k, r)}<\frac{\varepsilon}{3}+\frac{\varepsilon}{3}+\frac{\varepsilon}{3}=\varepsilon .
\end{aligned}
$$

Therefore, the $D^{k} f$ is continuous on $G$.

Remark 1 During the proof of Proposition 1 we have established the Kolmogorov type inequality between the uniform norm of fractional derivative of the function, the function itself and the norm of its higher-order derivative in the ideal lattice:

$$
\left\|D_{-}^{k} f\right\|_{L_{\infty}(G)} \leq \frac{2^{r}\|f\|_{L_{\infty}(G)}}{k h^{k} \varkappa(k, r)}+\left\|\chi_{(0, r h)}(\cdot) \int_{(\cdot) / r}^{h} \frac{N_{r}((\cdot) / t)}{t^{k+2-r}} d t\right\|_{E^{1}} \cdot\left\|f^{(r)}\right\|_{E^{*}}
$$

Proof of Proposition 2 First we note that

$$
\begin{aligned}
\int_{0}^{r} \frac{N_{r}(u)}{u^{r-k}} d u & =\frac{(-1)^{r}}{k(k-1) \cdots(k-r+1)}\left(\Delta_{-1}^{r}(\cdot)^{k}\right)(0) \\
& =\frac{(-1)^{r}}{k(k-1) \cdots(k-r+1)} \sum_{m=0}^{r}(-1)^{m}\left(\begin{array}{c}
r \\
m
\end{array}\right) m^{k} \\
& =\frac{\varkappa(k, r)}{\Gamma(-k)(-k)(-k+1) \cdots(-k+r-1)}=\frac{\varkappa(k, r)}{\Gamma(r-k)} .
\end{aligned}
$$


Let $f \in L_{\infty, E}^{r}(G)$ and $x \in G$. The derivative $D_{-}^{k} f(x)$ exists due to Proposition 1. Altering the order of integration and applying the Tonelli theorem we obtain

$$
\begin{aligned}
D_{-}^{k} f(x) & =\frac{(-1)^{r}}{\varkappa(k, r)} \int_{0}^{+\infty} \int_{0}^{r} t^{r-1-k} N_{r}(u) f^{(r)}(x+u t) d u d t \\
& =\frac{(-1)^{r}}{\varkappa(k, r)} \int_{0}^{+\infty} w^{r-k-1} f^{(r)}(x+w)\left(\int_{0}^{r} \frac{N_{r}(u)}{u^{r-k}} d u\right) d w \\
& =\frac{(-1)^{r}}{\Gamma(r-k)} \int_{0}^{+\infty} w^{r-1-k} f^{(r)}(x+w) d w
\end{aligned}
$$

which finishes the proof.

Proof of Proposition 3 Let $f \in L_{F, E}^{r}(G)$ and $x \in G$. Using the same arguments as in the proof of Proposition 1 we can prove that the first of two integrals

$$
\int_{0}^{1} \frac{\left(\Delta_{-t}^{r} f\right)(x)}{t^{1+k}} d t \text { and } \int_{1}^{+\infty} \frac{\left(\Delta_{-t}^{r} f\right)(x)}{t^{1+k}} d t
$$

is convergent. Hence, it is sufficient to prove the convergence of the second integral. The latter is obvious because

$$
\begin{aligned}
\left|\int_{1}^{+\infty} \frac{\left(\Delta_{-t}^{r} f\right)(x)}{t^{1+k}} d t\right| & \leq \sum_{m=0}^{r}\left(\begin{array}{c}
r \\
m
\end{array}\right) \int_{1}^{+\infty} \frac{|f(x+m t)|}{t^{1+k}} d t \\
& =\frac{|f(x)|}{k}+\sum_{m=1}^{r} m^{k}\left(\begin{array}{c}
r \\
m
\end{array}\right) \int_{m}^{+\infty} \frac{|f(x+u)|}{u^{1+k}} d u \\
& \leq \frac{|f(x)|}{k}+\sum_{m=1}^{r} m^{k}\left(\begin{array}{c}
r \\
m
\end{array}\right)\|f\|_{E} \cdot\left\|(\cdot)^{-1-k} \chi_{(m,+\infty)}(\cdot)\right\|_{F^{1}},
\end{aligned}
$$

and, for $m=1,2, \ldots, r$,

$$
\left\|(\cdot)^{-1-k} \chi_{(m,+\infty)}(\cdot)\right\|_{F^{1}} \leq \sum_{j=m}^{\infty} \frac{\left\|\chi_{(0,1)}\right\|_{F^{1}}}{j^{k+1}}<\frac{m+k}{m^{k+1} k}\left\|\chi_{(0,1)}\right\|_{F^{1}}
$$

Hence, $D_{-}^{k} f(x)$ exists, for every $x \in G$. Finally, we remark that equality (14) immediately holds true if $D_{-}^{k} f(x)$ exists. The proof is finished.

\section{Main results}

Let us present results on some general sufficient conditions allowing one to write a sharp Kolmogorov type inequality in various situations. We start with the Kolmogorov type inequality between the uniform norms of the Marchaud fractional derivative of a function, the function itself and its higher-order derivative. In Section 3.2 we extend this result on the case of inequalities between the norms of the function and its derivatives in an ideal lattice. Then in Section 3.3 we give another extension of results of Section 3.1 on the case of inequalities between the uniform norms of the Marchaud fractional derivative of a function, the uniform norm of the function itself, and the norm of the higher-order derivative in an ideal lattice. 
The idea used to prove main results of the paper consists in approximating fractional differentiation operator with the help of linear bounded operators. More precisely, let $G=$ $\mathbb{R}$ or $G=\mathbb{R}_{+}, X, Y, Z$ be the linear normed spaces of measurable on $G$ functions $f: G \rightarrow$ $\mathbb{R}, L \neq \emptyset$ be the space of the functions $f \in X$ having locally absolutely continuous on $G$ derivative of order $(r-1)$, and such that $f^{(r)} \in Z$. Let also $T: X \rightarrow Y$ and $R: Z \rightarrow Y$ be linear bounded operators such that, for every $f \in L, D_{-}^{k} f=T f+R f^{(r)}$. Then, for every $f \in L$,

$$
\left\|D_{-}^{k} f\right\|_{Y} \leq\|T f\|_{Y}+\left\|R f^{(r)}\right\|_{Y} \leq\|T\|_{X \rightarrow Y}\|f\|_{X}+\|R\|_{Z \rightarrow Y}\left\|f^{(r)}\right\|_{Z}
$$

which is an additive form of the Kolmogorov type inequality (7). If for some operators $T$ and $R$ there exists a function $f \in W$ turning the above inequality into an equality then the corresponding Kolmogorov type inequality is sharp.

We remark that this idea is not new and is already contained in [23] by Stechkin. Besides, some similar ideas were even in the papers by Landau and Hadamard. The corresponding operators $T$ and $R$ as well as the extremal function $f$ were found in many cases (see [28$30]$, and surveys $[1,2,4]$ for more details).

Let $G=\mathbb{R}$ or $G=\mathbb{R}_{+}$. By $V(G)$ we denote the space of the functions $f \in L_{1}(G)$ with bounded on $G$ variation. Also, we set $x_{+}:=\max \{x ; 0\}$, for every $x \in G$, and for $f \in L_{1}(G)$ and $m \in \mathbb{N}$, we denote by $f^{[m]}$ the $m$ th-order integral of the function $f$ :

$$
f^{[m]}(x):=\frac{1}{(m-1) !} \int_{G}(x-t)_{+}^{m-1} f(t) d t, \quad x \in G
$$

Finally, for $\tau>0$, we define the function $\mathcal{R}_{\tau}: G \rightarrow \mathbb{R}$ as follows:

$$
\mathcal{R}_{\tau}(x)= \begin{cases}\frac{x^{\tau-1}}{\Gamma(\tau)}, & x>0 \\ 0, & x \leq 0\end{cases}
$$

\subsection{The Kolmogorov type inequalities for the Marchaud fractional derivatives: case of uniform norms}

The following results hold true.

Theorem 1 Let $G=\mathbb{R}_{+}$or $G=\mathbb{R}, r \in \mathbb{N}, k \in(0, r) \backslash \mathbb{N}$, and a function $\Omega \in V(G)$ be such that $\left(\mathcal{R}_{r-k}-\Omega^{[r-1]}\right) \in L_{1}(G)$ and, for every $f \in L_{\infty, \infty}^{r}(G)$,

$$
D_{-}^{k} f(0)-\int_{G} f(x) d \Omega(x)=(-1)^{r} \int_{G}\left(\mathcal{R}_{r-k}(x)-\Omega^{[r-1]}(x)\right) f^{(r)}(x) d x .
$$

Then, for every $f \in L_{\infty, \infty}^{r}(G)$ and $h>0$, we have the inequality

$$
\begin{aligned}
\left\|D_{-}^{k} f\right\|_{L_{\infty}(G)} \leq & h^{-k} \bigvee_{G} \Omega \cdot\|f\|_{L_{\infty}(G)} \\
& +h^{r-k}\left\|\mathcal{R}_{r-k}-\Omega^{[r-1]}\right\|_{L_{1}(G)} \cdot\left\|f^{(r)}\right\|_{L_{\infty}(G)} .
\end{aligned}
$$

Furthermore, if a function $\Phi \in W_{\infty, \infty}^{r}(G)$ satisfies the equalities

$$
\int_{G} \Phi(t) d \Omega(t)=\bigvee_{G} \Omega \cdot\|\Phi\|_{L_{\infty}(G)}
$$


and

$$
(-1)^{r} \int_{G}\left(\mathcal{R}_{r-k}(x)-\Omega^{[r-1]}(x)\right) \Phi^{(r)}(x) d x=\left\|\mathcal{R}_{r-k}-\Omega^{[r-1]}\right\|_{L_{1}(G)}
$$

then (18) is sharp and the function $\Phi_{h}(\cdot):=\Phi((\cdot) / h)$ turns (18) into an equality.

Minimizing the right hand part of (18) by $h$ we obtain the next consequence.

Corollary 1 Let $G=\mathbb{R}_{+}$or $G=\mathbb{R}, r \in \mathbb{N}, k \in(0, r) \backslash \mathbb{N}$, and assume that the functions $\Omega$ and $\Phi$ satisfy assumptions of Theorem 1 . Then, for every $f \in L_{\infty, \infty}^{r}(G)$, the following sharp inequality holds true:

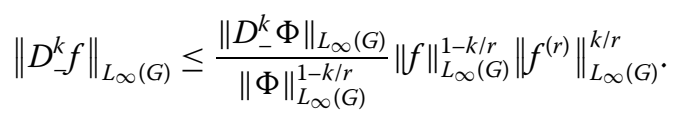

We remark that the following results on sharp inequalities of the form (7) concretize Corollary 1.

1. For $G=\mathbb{R}, r=2$, and $k \in(0,1)$, the extremal function $\Phi$ in inequality (18) and the corresponding function $\Omega$ that satisfy the conditions of Corollary 1 were found by Geĭsberg [8] and Arestov [10], respectively:

$$
\Omega(x):=\left\{\begin{array}{ll}
0, & x \leq 0, \\
\frac{1}{\Gamma(2-k)}, & x \in(0,1), \\
\frac{x^{-k}}{\Gamma(1-k)}, & x \geq 1,
\end{array} \quad \Phi(x):= \begin{cases}-\frac{(1+p)^{2}}{8}, & x \leq-p, \\
\frac{(x+p)^{2}-(1+p)^{2}}{8}, & x \in\left[-p, \frac{1-p}{2}\right] \\
\frac{(1+p)^{2}-(1-x)^{2}}{8}, & x \in\left[\frac{1-p}{2}, 1\right] \\
\frac{(1+p)^{2}}{8}, & x \geq 1,\end{cases}\right.
$$

where $p=1-2^{k /(1-k)}$.

2. For $G=\mathbb{R}_{+}, r=2$, and $k \in(0,1)$, the extremal function $\Phi$, and the corresponding function $\Omega$ that satisfy the conditions of Corollary 1 were found by Arestov [10, Theorem 3]:

$$
\Omega(x):=\left\{\begin{array}{ll}
0, & x=0, \\
\frac{1}{\Gamma(2-k)}, & x \in(0,1), \\
\frac{x^{-k}}{\Gamma(1-k)}, & x \geq 1,
\end{array} \quad \Phi(x):= \begin{cases}\frac{1}{4}-x+\frac{x^{2}}{2}, & x \in[0,1] \\
-\frac{1}{4}, & x \geq 1 .\end{cases}\right.
$$

3. For $G=\mathbb{R}_{+}, r=2$, and $k \in(1,2)$, the extremal function $\Phi$ and the corresponding function $\Omega$ that satisfy the conditions of Corollary 1 were also found by Arestov [10, p.32]:

$$
\begin{aligned}
& \Omega(x):= \begin{cases}0, & x=0, \\
\frac{3-2^{(k+1) / 2}}{\Gamma(2-k)}, & x \in(0, \sqrt{2}-1], \\
\frac{2^{k / 2}-\sqrt{2}}{\Gamma(2-k)(\sqrt{2}-1)}, & x \in(\sqrt{2}-1,1), \\
\frac{x^{-k}}{\Gamma(1-k)}, & x \geq 1,\end{cases} \\
& \Phi(x):= \begin{cases}\frac{3-2 \sqrt{2}-4(\sqrt{2}-1) x+2 x^{2}}{4}, & x \in\left[0, \frac{1}{\sqrt{2}}\right], \\
\frac{1-2 \sqrt{2}+4 x-2 x^{2}}{4}, & x \in\left(\frac{1}{\sqrt{2}}, 1\right), \\
\frac{3-2 \sqrt{2}}{4}, & x \geq 1 .\end{cases}
\end{aligned}
$$


For integer values of $k$, the extremal function $\Phi$ on $\mathbb{R}$ in inequality (21) was found by Kolmogorov [31] (see also [3]), for every $r=2,3, \ldots$ In the surveys [1, 2, 4] the reader could find more references and a detailed history of the subject and overview of cases when the extremal function $\Phi$ in inequality (21) on $\mathbb{R}_{+}$is known.

In addition, for integer values of $k$, the function $\Omega$ on $\mathbb{R}_{+}$for which inequality (18) is sharp was explicitly constructed by Stechkin [23] in the case $r=2,3$. In the case $G=\mathbb{R}$ the existence of such a function $\Omega$ was proved by Domar [32] and explicitly it was constructed by Stechkin [23] for $r=2,3$, Arestov [33] for $r=4,5$, and Buslaev [34] for $r>5$.

Proof of Theorem 1 First, we let $h=1$ and define the linear operator $T: L_{\infty}(G) \rightarrow L_{\infty}(G)$ as follows:

$$
\operatorname{Tg}(\cdot):=\int_{G} g(\cdot+t) d \Omega(t), \quad g \in L_{\infty}(G)
$$

Clearly, $T$ is bounded and $\|T\|=\bigvee_{G} \Omega$. Next, let a function $f \in L_{\infty, \infty}^{r}(G)$ and a point $x \in G$ be arbitrary. Then from Proposition 2 and (17) we deduce

$$
\begin{aligned}
\left|D_{-}^{k} f(x)\right| & =\left|T f(x)+\left((-1)^{r} \int_{G} \mathcal{R}_{r-k}(t) f^{(r)}(x+t) d t-T f(x)\right)\right| \\
& \leq|T f(x)|+\left|(-1)^{r} \int_{G}\left(\mathcal{R}_{r-k}(t)-\Omega^{[r-1]}(t)\right) f^{(r)}(x+t) d t\right| \\
& \leq \bigvee_{G} \Omega \cdot\|f\|_{L_{\infty}(G)}+\left\|\mathcal{R}_{r-k}-\Omega^{[r-1]}\right\|_{L_{1}(G)} \cdot\left\|f^{(r)}\right\|_{L_{\infty}(G)},
\end{aligned}
$$

which implies the desired inequality (18) in the case $h=1$ :

$$
\left\|D_{-}^{k} f\right\|_{L_{\infty}(G)} \leq \bigvee_{G} \Omega \cdot\|f\|_{L_{\infty}(G)}+\left\|\mathcal{R}_{r-k}-\Omega^{[r-1]}\right\|_{L_{1}(G)} \cdot\left\|f^{(r)}\right\|_{L_{\infty}(G)} \cdot
$$

Next, we assume that there exists a function $\Phi \in W_{\infty, \infty}^{r}(G)$ satisfying equalities (19) and (20). Due to Proposition 1 the derivative $D_{-}^{k} \Phi$ is continuous on $G$. Hence, taking into account equalities (19) and (20) we have

$$
\begin{aligned}
\left\|D_{-}^{k} \Phi\right\|_{L_{\infty}(G)} & \geq\left|D_{-}^{k} \Phi(0)\right| \\
& =\left|\int_{G} \Phi(x) d \Omega(x)+(-1)^{r} \int_{G}\left(\mathcal{R}_{r-k}(x)-\Omega^{[r-1]}(x)\right) \Phi^{(r)}(x) d x\right| \\
& \geq \bigvee_{G} \Omega \cdot\|\Phi\|_{L_{\infty}(G)}+\left\|\mathcal{R}_{r-k}-\Omega^{[r-1]}\right\|_{L_{1}(G)} \cdot\left\|\Phi^{(r)}\right\|_{L_{\infty}(G)} .
\end{aligned}
$$

Therefore, the statement of the theorem is proved in the case $h=1$.

Now, we let $h>0$ and $f \in L_{\infty, \infty}^{r}(G)$ be arbitrary, and consider the function $f_{h}(x):=f(x / h)$, $x \in G$. Evidently, $f_{h} \in L_{\infty, \infty}^{r}(G)$ and by substituting $f_{h}$ into (22) we derive inequality (18). Clearly, $\Phi_{h}$ turns (18) into an equality. 


\subsection{The Kolmogorov type inequalities for the Marchaud fractional derivatives: case of norms in an ideal lattice}

Let us generalize Theorem 1 to the case of Kolmogorov type inequalities between the norms of the Marchaud fractional derivative of a function, the function itself, and its higher-order derivative in an ideal lattice.

Theorem 2 Let $G=\mathbb{R}$ or $G=\mathbb{R}_{+}$, E be a semi shift-invariant lattice on $G, r \in \mathbb{N}$, and $k \in(0, r) \backslash \mathbb{N}$. Let also $\Omega \in V(G)$ be such that $\left(\mathcal{R}_{r-k}-\Omega^{[r-1]}\right) \in L_{1}(G)$ and (17) hold true, for every $f \in L_{E, E}^{r}(G)$. Then, for every $f \in L_{E, E}^{r}(G)$,

$$
\left\|D_{-}^{k} f\right\|_{E} \leq \bigvee_{G} \Omega \cdot\|f\|_{E}+\left\|\mathcal{R}_{r-k}-\Omega^{[r-1]}\right\|_{L_{1}(G)} \cdot\left\|f^{(r)}\right\|_{E}
$$

An immediate consequence of Theorem 2 is the following.

Corollary 2 Let $G=\mathbb{R}$ or $G=\mathbb{R}_{+}, 1 \leq s \leq \infty, r \in \mathbb{N}$, and $k \in(0, r) \backslash \mathbb{N}$. Let also a function $\Omega \in V(G)$ be such that $\left(\mathcal{R}_{r-k}-\Omega^{[r-1]}\right) \in L_{1}(G)$ and (17) holds true, for every $f \in L_{s, s}^{r}(G)$. Then, for every $f \in L_{s, s}^{r}(G)$ and $h>0$,

$$
\left\|D_{-}^{k} f\right\|_{L_{s}(G)} \leq h^{-k} \bigvee_{G} \Omega \cdot\|f\|_{L_{s}(G)}+h^{r-k}\left\|\mathcal{R}_{r-k}-\Omega^{[r-1]}\right\|_{L_{1}(G)} \cdot\left\|f^{(r)}\right\|_{L_{s}(G)} .
$$

Moreover, if a function $\Phi \in W_{\infty, \infty}^{r}(G)$ satisfies (19) and (20) then, for $f \in L_{s, s}^{r}(G)$,

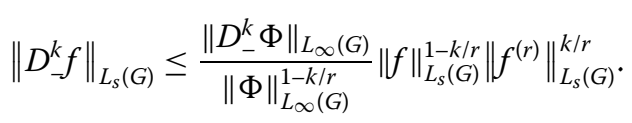

Evidently, inequality (23) is sharp for $s=\infty$. In Section 4.1 we shall show that this inequality is also sharp when $s=1, r=1$, and $G=\mathbb{R}$. For integer values of $k$ and $G=\mathbb{R}$, inequality (23) is known as the Stein inequality [35] (see also [36, 37]).

Proof of Theorem 2 Using Proposition 3 and the generalized Minkowskii inequality (see [18]), for every function $f \in L_{E, E}^{r}(G)$, we have

$$
\begin{aligned}
\left\|D_{-}^{k} f\right\|_{E} & \leq\left\|\int_{G} f(x) d \Omega(x)\right\|_{E}+\left\|\int_{G}\left(\mathcal{R}_{r-k}(x)-\Omega^{[r-1]}(x)\right) f^{(r)}(x) d x\right\|_{E} \\
& \leq \bigvee_{G} \Omega \cdot\|f\|_{E}+\left\|\mathcal{R}_{r-k}-\Omega^{[r-1]}\right\|_{L_{1}(G)} \cdot\left\|f^{(r)}\right\|_{E} .
\end{aligned}
$$

The proof is finished.

\subsection{The Kolmogorov type inequalities for the Marchaud fractional derivatives: case when the norm of the higher-order derivative is considered in an ideal lattice}

In this subsection we generalize the results of Section 3.1 on the case when the norm of the higher-order derivative is taken in an ideal lattice. For convenience, we split the subsection into two parts: first we present results concerning the case when extremal function in the Kolmogorov type inequality (i.e. turning it into an equality) exists and then we present 
results concerning the case when the extremal function in the Kolmogorov type inequality does not exist. For integral-order derivatives, the existence of an extremal function in the Kolmogorov type inequalities (1) was proved in the case when $1 \leq p, q \leq \infty, 1<s \leq \infty$, and inequality (3) is strict. For the corresponding results, we refer the reader to $[24,38$, 39].

3.3.1 Case of existence of extremal function in the Kolmogorov type inequality For an ideal lattice $E$ on $G$ and $r \in \mathbb{N}$, we set

$$
W_{\infty, E}^{r}(G)=\left\{f \in L_{\infty, E}^{r}(G):\left\|f^{(r)}\right\|_{E} \leq 1\right\}
$$

Theorem 3 Let $G=\mathbb{R}_{+}$or $G=\mathbb{R}, r \in \mathbb{N}, k \in(0, r) \backslash \mathbb{N}, E$ be an ideal semi shift-invariant lattice on $G$ satisfying conditions (12) and (13), and $E^{1}$ be the associated space to E. Also, let a function $\Omega \in V(G)$ be such that $\left(\mathcal{R}_{r-k}-\Omega^{[r-1]}\right) \in E^{1}$ and (17) hold true, for every $f \in$ $L_{\infty, E}^{r}(G)$. Then, for every $f \in L_{\infty, E}^{r}(G)$,

$$
\left\|D_{-}^{k} f\right\|_{L_{\infty}(G)} \leq \bigvee_{G} \Omega \cdot\|f\|_{L_{\infty}(G)}+\left\|\mathcal{R}_{r-k}-\Omega^{[r-1]}\right\|_{E^{1}} \cdot\left\|f^{(r)}\right\|_{E^{\prime}}
$$

Furthermore, if a function $\Phi \in W_{\infty, E}^{r}(G)$ satisfies equalities (19) and

$$
(-1)^{r} \int_{G}\left(\mathcal{R}_{r-k}(x)-\Omega^{[r-1]}(x)\right) \Phi^{(r)}(x) d x=\left\|\mathcal{R}_{r-k}-\Omega^{[r-1]}\right\|_{E^{1}},
$$

then inequality (24) is sharp and $\Phi$ turns (24) into an equality.

We remark that Theorem 3 can be generalized as follows.

Theorem 4 Let $G=\mathbb{R}$ or $G=\mathbb{R}_{+}, r \in \mathbb{N}, k \in(0, r) \backslash \mathbb{N}, E$ be a semi-shift invariant lattice on $G$ that satisfy conditions (13), $E^{1}$ be the associated space to $E, F$ be an ideal lattice such that its associated space $F^{1}$ contains the function $\chi_{(0,1)}$. Let also a locally absolutely continuous function $\Omega \in V(G)$ on $G$ be such that $\left(\mathcal{R}_{r-k}-\Omega^{[r-1]}\right) \in E^{1}$ and (17) holds true, for every $f \in L_{F, E}^{r}(G)$. Then, for every $f \in L_{F, E}^{r}(G)$,

$$
\left\|D_{-}^{k} f\right\|_{L_{\infty}(G)} \leq\left\|\Omega^{\prime}\right\|_{F^{1}} \cdot\|f\|_{F}+\left\|\mathcal{R}_{r-k}-\Omega^{[r-1]}\right\|_{E^{1}} \cdot\left\|f^{(r)}\right\|_{E^{*}}
$$

For the spaces $L_{s}(G), 1<s \leq \infty$, we obtain the following consequence.

Corollary 3 Let $G=\mathbb{R}$ or $G=\mathbb{R}_{+}, 1<s \leq \infty, s^{\prime}=s /(s-1), r \in \mathbb{N}$, and $k \in(0, r-1 / s) \backslash \mathbb{N}$. Let also a function $\Omega \in V(G)$ be such that $\left(\mathcal{R}_{r-k}-\Omega^{[r-1]}\right) \in L_{s^{\prime}}(G)$ and (17) holds true, for every $f \in L_{\infty, s}^{r}(G)$. If a function $\Phi \in W_{\infty, s}^{r}(G)$ satisfies equality (19) and the relation

$$
(-1)^{r} \int_{G}\left(\mathcal{R}_{r-k}(x)-\Omega^{[r-1]}(x)\right) \Phi^{(r)}(x) d x=\left\|\mathcal{R}_{r-k}-\Omega^{[r-1]}\right\|_{L_{s^{\prime}}(G)}
$$

then, for every $f \in L_{\infty, s}^{r}(G)$ and $h>0$, the sharp inequalities

$$
\begin{aligned}
\left\|D_{-}^{k} f\right\|_{L_{\infty}(G)} \leq & h^{-k} \bigvee_{G} \Omega \cdot\|f\|_{L_{\infty}(G)} \\
& +h^{r-k-1 / s}\left\|\mathcal{R}_{r-k}-\Omega^{[r-1]}\right\|_{L_{s^{\prime}}(G)} \cdot\left\|f^{(r)}\right\|_{L_{s}(G)}
\end{aligned}
$$


and

$$
\left\|D_{-}^{k} f\right\|_{L_{\infty}(G)} \leq \frac{\left\|D_{-}^{k} \Phi\right\|_{L_{\infty}(G)}}{\|\Phi\|_{L_{\infty}(G)}^{1-\lambda}}\|f\|_{L_{\infty}(G)}^{1-\lambda}\left\|f^{(r)}\right\|_{L_{S}(G)}^{\lambda}, \quad \lambda=\frac{k}{r-1 / s}
$$

hold true. Moreover, the function $\Phi_{h}(\cdot):=h^{r-1 / s} \Phi((\cdot) / h)$ turns (26) and (27) into equalities.

We remark that Theorems 3.1.2 and 3.2.2 [16] are concretizations of Corollary 3. In addition, in the case of integer values of $k$ and $r=2,3$ and $1<s<\infty$, the functions $\Phi$ and $\Omega$ satisfying conditions of Corollary 26 were explicitly constructed by Arestov in [28].

Proof of Theorem 3 The proof is similar to the proof of Theorem 1 in the case $h=1$. The difference is that for a function $f \in L_{\infty, E}^{r}(G)$ and $x \in G$, we need to use the inequality

$$
\begin{aligned}
& \left|\int_{G}\left(\mathcal{R}_{r-k}(t)-\Omega^{[r-1]}(t)\right) f^{(r)}(x+t) d t\right| \\
& \quad \leq\left\|\mathcal{R}_{r-k}-\Omega^{[r-1]}\right\|_{E^{1}} \cdot\left\|f^{(r)}(x+\cdot)\right\|_{E} \leq\left\|\mathcal{R}_{r-k}-\Omega^{[r-1]}\right\|_{E^{1}} \cdot\left\|f^{(r)}\right\|_{E} .
\end{aligned}
$$

The extremity of the function $\Phi$ can be proved in a similar way to Theorem 1 .

Proof of Corollary 3 For every $h>0$, we observe that the functions $\Omega_{h}(x):=h^{-k} \Omega(x / h)$, $x \in G$, and $\Phi_{h}$ satisfy conditions (17), (19), and (25). Moreover,

$$
\bigvee_{G} \Omega_{h}=h^{-k} \bigvee_{G} \Omega, \quad\left\|\mathcal{R}_{r-k}-\Omega_{h}^{[r-1]}\right\|_{L_{s^{\prime}}(G)}=h^{r-k-1 / s}\left\|\mathcal{R}_{r-k}-\Omega^{[r-1]}\right\|_{L_{s^{\prime}}(G)}
$$

Hence, by Theorem 3 the desired inequality (26) holds true and the function $\Phi_{h}$ turns (26) into an equality. Finally, minimizing the right hand part of (26) by the variable $h$, we arrive at inequality (27). The proof is finished.

\subsubsection{Case of non-existence of extremal function in the Kolmogorov type inequality}

Let us present two results showing when conditions (19) and (25) can be relaxed.

Theorem 5 Let $G=\mathbb{R}_{+}$or $G=\mathbb{R}$, and numbers $k, r$, an ideal semi shift-invariant lattice $E$ on $G$ and a function $\Omega \in V(G)$ satisfy assumptions of Theorem 3. Also, assume that there is a family of the functions $\left\{\Phi_{\varepsilon}\right\}_{\varepsilon>0} \subset W_{\infty, E}^{r}(G)$ satisfying the equality (19) and, for $\varepsilon>0$, the inequality

$$
(-1)^{r} \int_{G}\left(\mathcal{R}_{r-k}(x)-\Omega^{[r-1]}(x)\right) \Phi_{\varepsilon}^{(r)}(x) d x>\left\|\mathcal{R}_{r-k}-\Omega^{[r-1]}\right\|_{E^{1}}-\varepsilon .
$$

Then inequality (24) holds true and is sharp in the sense that, for every sufficiently small $\varepsilon>0$, there exists a function $f_{\varepsilon} \in L_{\infty, E}^{r}(G)$ such that

$$
\left\|D_{-}^{k} f_{\varepsilon}\right\|_{L_{\infty}(G)}>\bigvee_{G} \Omega \cdot\left\|f_{\varepsilon}\right\|_{L_{\infty}(G)}+\left(\left\|\mathcal{R}_{r-k}-\Omega^{[r-1]}\right\|_{E^{1}}-\varepsilon\right) \cdot\left\|f_{\varepsilon}^{(r)}\right\|_{E^{*}}
$$


Theorem 6 Let $G=\mathbb{R}_{+}$or $G=\mathbb{R}, r \in \mathbb{N}, k \in(0, r-1) \backslash \mathbb{N}, E$ be an ideal semi shiftinvariant lattice on $G$ such that $\liminf _{h \rightarrow 0^{+}}\left(h^{-1}\left\|\chi_{(0, h)}\right\|_{E}\right)=: \mu \in(0,+\infty)$. Also, let a function $\Omega \in V(G)$ be such that $\left(\mathcal{R}_{r-k}-\Omega^{[r-1]}\right) \in E^{1}$ and (17) holds true, for every $f \in L_{\infty, E}^{r}(G)$. Assume that there exists a function $\Phi \in L_{\infty}(G)$ such that its derivative $\Phi^{(r-1)}$ is piecewise constant on $G, \bigvee_{G} \Phi^{(r-1)}=\mu^{-1}$, there exists $h_{0}>0$ such that the distance between each pair of discontinuity points of $\Phi^{(r-1)}$ is bounded below by $h_{0}$, and equalities (19) and

$$
(-1)^{r} \int_{G}\left(\mathcal{R}_{r-k}(x)-\Omega^{[r-1]}(x)\right) d \Phi^{(r-1)}(x)=\left\|\mathcal{R}_{r-k}-\Omega^{[r-1]}\right\|_{E^{1}}
$$

are valid. Then inequality (24) holds true and is sharp.

In the case $E=L_{1}(G)$ we obtain the following.

Corollary 4 Let $G=\mathbb{R}$ or $G=\mathbb{R}_{+}, r \in \mathbb{N}$ and $k \in(0, r-1) \backslash \mathbb{N}$. Let also a function $\Omega \in$ $V(G)$ be such that $\left(\mathcal{R}_{r-k}-\Omega^{[r-1]}\right) \in L_{\infty}(G)$ and (17) holds true, for every $f \in L_{\infty, 1}^{r}(G)$. If an $(r-1)$-times differentiable function $\Phi$ with piecewise constant derivative $\Phi^{(r-1)}$ satisfies equalities (19), $\bigvee_{G} \Phi^{(r-1)}=1$, there exists $h_{0}>0$ such that the distance between each pair of discontinuity points of $\Phi^{(r-1)}$ is bounded below by $h_{0}$, and

$$
(-1)^{r} \int_{G}\left(\mathcal{R}_{r-k}(x)-\Omega^{[r-1]}(x)\right) d \Phi^{(r-1)}(x)=\left\|\mathcal{R}_{r-k}-\Omega^{[r-1]}\right\|_{L_{\infty}(G)}
$$

then, for every $f \in L_{\infty, 1}^{r}(G)$ and $h>0$, the sharp inequalities

$$
\left\|D_{-}^{k} f\right\|_{L_{\infty}(G)} \leq h^{-k} \bigvee_{G} \Omega \cdot\|f\|_{L_{\infty}(G)}+h^{r-k-1}\left\|\mathcal{R}_{r-k}-\Omega^{[r-1]}\right\|_{L_{\infty}(G)} \cdot\left\|f^{(r)}\right\|_{L_{1}(G)}
$$

and

$$
\left\|D_{-}^{k} f\right\|_{L_{\infty}(G)} \leq \frac{\left\|D_{-}^{k} \Phi\right\|_{L_{\infty}(G)}}{\|\Phi\|_{L_{\infty}(G)}^{1-\lambda}}\|f\|_{L_{\infty}(G)}^{1-\lambda}\left\|f^{(r)}\right\|_{L_{1}(G)}^{\lambda}, \quad \lambda=\frac{k}{r-1},
$$

hold true.

We remark that for integer values of $k$ and $r=2,3$, the functions $\Phi$ and $\Omega$ satisfying conditions of Corollary 4 were constructed by Arestov in [28].

Proof of Theorem 5 We observe that inequality (24) holds true, for every $f \in L_{\infty, E}^{r}(G)$. Let us prove that (24) is sharp. Let $\varepsilon>0$ be arbitrary and sufficiently small. Due to Proposition 1 the fractional derivative $D_{-}^{k} \Phi_{\varepsilon}$ is continuous on $G$. Hence, taking into account equalities (19) and (28) we obtain

$$
\begin{aligned}
\left\|D_{-}^{k} \Phi_{\varepsilon}\right\|_{L_{\infty}(G)} & \geq\left|D_{-}^{k} \Phi_{\varepsilon}(0)\right| \\
& =\left|\int_{G} \Phi_{\varepsilon}(x) d \Omega(x)+(-1)^{r} \int_{G}\left(\mathcal{R}_{r-k}(x)-\Omega^{[r-1]}(x)\right) \Phi_{\varepsilon}^{(r)}(x) d x\right| \\
& \geq \bigvee_{G} \Omega \cdot\left\|\Phi_{\varepsilon}\right\|_{L_{\infty}(G)}+\left\|\mathcal{R}_{r-k}-\Omega^{[r-1]}\right\|_{E^{1}}-\varepsilon \\
& \geq \bigvee_{G} \Omega \cdot\left\|\Phi_{\varepsilon}\right\|_{L_{\infty}(G)}+\left(\left\|\mathcal{R}_{r-k}-\Omega^{[r-1]}\right\|_{E^{1}}-\varepsilon\right) \cdot\left\|\Phi_{\varepsilon}^{(r)}\right\|_{E^{\prime}}
\end{aligned}
$$

The proof is finished. 
Proof of Theorem 6 Since $k<r-1$ we see that $(\cdot)^{r-k-1} \chi_{(0,1)}(\cdot) \in E^{1}$ and condition (13) is also fulfilled. Hence, by Theorem 3, inequality (24) holds true. Let us prove that inequality (24) is sharp. To this end by $B=\left\{\beta_{j}\right\}_{j \in J}$ ( $J$ is a finite or countable set of indices) we denote the discontinuity points of $\Phi^{(r-1)}$ and set $\alpha_{j}:=\Phi\left(\beta_{j}^{+}\right)-\Phi\left(\beta_{j}^{-}\right), j \in J$. Due to the assumption there exists $h_{0}>0$ such that $\left|\beta_{j}-\beta_{i}\right| \geq h_{0}$ for all distinct indices $i, j \in J$. Now, for every $h \in\left(0, h_{0}\right)$, we define the function

$$
\Phi_{h}(x):=\frac{1}{h} \int_{0}^{h} \Phi(x+t) d t, \quad x \in G
$$

It is easy to show that as $h \rightarrow 0^{+}$, we have

$$
\begin{aligned}
& \int_{G} \Phi_{h}(x) d \Omega(x) \rightarrow \int_{G} \Phi(x) d \Omega(x)=\bigvee_{G} \Omega \cdot\|\Phi\|_{L_{\infty}(G)} \\
& \int_{G}\left(\mathcal{R}_{r-k}(x)-\Omega^{[r-1]}(x)\right) \Phi_{h}^{(r)}(x) d x \rightarrow \int_{G}\left(\mathcal{R}_{r-k}(x)-\Omega^{[r-1]}(x)\right) d \Phi^{(r-1)}(x) \\
& \quad=(-1)^{r}\left\|\mathcal{R}_{r-k}-\Omega^{[r-1]}\right\|_{E^{1}},
\end{aligned}
$$

and

$$
\liminf _{h \rightarrow 0^{+}}\left\|\Phi_{h}^{(r)}\right\|_{E} \leq \liminf _{h \rightarrow 0^{+}} \sum_{j \in J} \frac{\left|\alpha_{j}\right|}{h} \cdot\left\|\chi_{(0, h)}\right\|_{E}=\frac{1}{\mu} \cdot \liminf _{h \rightarrow 0^{+}} \frac{\left\|\chi_{(0, h)}\right\|_{E}}{h}=1 .
$$

Due to the continuity of $D_{-}^{k} \Phi_{h}$, for every $\varepsilon$ and every sufficiently small $h>0$,

$$
\begin{aligned}
\left\|D_{-}^{k} \Phi_{h}\right\|_{L_{\infty}(G)} & \geq D_{-}^{k} \Phi_{h}(0)>\bigvee_{G} \Omega \cdot\left\|\Phi_{h}\right\|_{L_{\infty}(G)}+\left\|\mathcal{R}_{r-k}-\Omega^{[r-1]}\right\|_{E^{1}}-\varepsilon \\
& \geq \bigvee_{G} \Omega \cdot\left\|\Phi_{h}\right\|_{L_{\infty}(G)}+\left(\left\|\mathcal{R}_{r-k}-\Omega^{[r-1]}\right\|_{E^{1}}-\varepsilon\right)\left\|\Phi_{h}^{(r)}\right\|_{E^{*}}
\end{aligned}
$$

The proof is finished.

\section{Consequences of main results}

In this section we deduce new sharp Kolmogorov type inequalities from the results of the previous section when the order of the higher-order derivative is 1 or 2 .

\subsection{Case $r=1$ and $k \in(0,1)$}

Let $G=\mathbb{R}$ or $G=\mathbb{R}_{+}$. For $k \in(0,1)$ and $h>0$, we set

$$
\tau_{h}(x):= \begin{cases}0, & x \notin G \backslash(0, h), \\ x^{-k}-h^{-k}, & x \in(0, h) .\end{cases}
$$

The following proposition is the consequence of Theorem 5.

Corollary 5 Let $G=\mathbb{R}$ or $G=\mathbb{R}_{+}, k \in(0,1)$, E be an ideal semi shift-invariant lattice on $G$ satisfying conditions (12) and (13), $E^{1}$ be the associated space to $E$. Then, for every $f \in$ 
$L_{\infty, E}^{1}(G)$ and $h>0$, the sharp inequality

$$
\left\|D_{-}^{k} f\right\|_{L_{\infty}(G)} \leq \frac{2 h^{-k}}{\Gamma(1-k)}\|f\|_{L_{\infty}(G)}+\frac{\left\|\tau_{h}\right\|_{E^{1}}}{\Gamma(1-k)}\left\|f^{\prime}\right\|_{E}
$$

holds true.

Proof For every $h>0$, we define the function

$$
\Omega(x):=\mathcal{R}_{1-k}(x)-\frac{\tau_{h}(x)}{\Gamma(1-k)}= \begin{cases}0, & x \in G \backslash(0,+\infty), \\ \frac{h^{-k}}{\Gamma(1-k)}, & x \in(0, h), \\ \frac{x^{-k}}{\Gamma(1-k)}, & x \geq h .\end{cases}
$$

It is easy to check that (17) holds true, for every $f \in L_{\infty, E}^{1}(G), \bigvee_{G} \Omega=\frac{2 h^{-k}}{\Gamma(1-k)}$, and $\mathcal{R}_{1-k}-\Omega=$ $\frac{\tau_{h}}{\Gamma(1-k)} \in E^{1}$.

Let us construct a family of functions $\left\{\Phi_{\varepsilon}\right\}_{\varepsilon>0} \subset W_{\infty, E}^{1}(G)$ satisfying conditions (19) and (28). For every $\varepsilon>0$, there exists a function $g_{\varepsilon} \in E,\left\|g_{\varepsilon}\right\|_{E} \leq 1$, such that

$$
\int_{0}^{h}\left(\mathcal{R}_{1-k}(x)-\Omega(x)\right) g_{\varepsilon}(x) d x>\left\|\mathcal{R}_{1-k}-\Omega\right\|_{E^{1}}-\varepsilon=\frac{\left\|\tau_{h}\right\|_{E^{1}}}{\Gamma(1-k)}-\varepsilon .
$$

Without loss of generality we may assume that $g_{\varepsilon}$ is non-negative on $G$ and $\operatorname{supp} g_{\varepsilon}=[0, h]$. Next, we define the function $\Phi_{\varepsilon}$ as the first integral of $\left(-g_{\varepsilon}\right)$ :

$$
\Phi_{\varepsilon}(x):=-\int_{0}^{x} g_{\varepsilon}(t) d t+\frac{1}{2} \int_{0}^{h} g_{\varepsilon}(t) d t, \quad x \in G .
$$

Clearly, $\Phi_{\varepsilon} \in W_{\infty, E}^{1}(G)$ and $\Phi_{\varepsilon}(x)=-\Phi_{\varepsilon}(0)=-\left\|\Phi_{\varepsilon}\right\|_{L_{\infty}(G)}, x \geq h$. As a result,

$$
\int_{G} \Phi_{\varepsilon}(x) d \Omega(x)=\frac{h^{-k} \Phi_{\varepsilon}(0)}{\Gamma(1-k)}+\int_{h}^{\infty} \frac{\Phi_{\varepsilon}(x) d\left(x^{-k}\right)}{\Gamma(1-k)}=\bigvee_{G} \Omega \cdot\left\|\Phi_{\varepsilon}\right\|_{L_{\infty}(G)}
$$

and

$$
-\int_{G}\left(\mathcal{R}_{1-k}(x)-\Omega(x)\right) \Phi_{\varepsilon}^{\prime}(x) d x=\int_{0}^{h}\left(\mathcal{R}_{1-k}(x)-\Omega(x)\right) g_{\varepsilon}(x) d x>\left\|\mathcal{R}_{1-k}-\Omega\right\|_{E^{1}}-\varepsilon .
$$

Therefore, the function $\Omega$ and the family of the functions $\left\{\Phi_{\varepsilon}\right\}_{\varepsilon>0}$ satisfy the assumptions of Theorem 5 . Hence, inequality (29) holds true and is sharp.

Next, we formulate the following Stein type inequality.

Corollary 6 For $k \in(0,1), h>0$, and $f \in L_{\infty, 1}^{1}(\mathbb{R})$, the sharp inequalities

$$
\begin{aligned}
\left\|D_{-}^{k} f\right\|_{L_{1}(\mathbb{R})} & \leq \frac{2 h^{-k}\|f\|_{L_{1}(\mathbb{R})}}{\Gamma(1-k)}+\frac{k h^{1-k}\left\|f^{\prime}\right\|_{L_{1}(\mathbb{R})}}{\Gamma(2-k)} \\
\left\|D_{-}^{k} f\right\|_{L_{1}(\mathbb{R})} & \leq \frac{2^{1-k}}{\Gamma(2-k)}\|f\|_{L_{1}(\mathbb{R})}^{1-k}\left\|f^{\prime}\right\|_{L_{1}(\mathbb{R})}^{k}
\end{aligned}
$$

hold true. 
Proof For $h>0$, let $\Omega$ be defined by (30). Then both desired inequalities follow from Corollary 2. Let us prove that inequalities (31) are sharp. To this end, for every $\varepsilon \in(0, h)$, we consider the Steklov averaging operator $S_{\varepsilon}: L_{\infty}(G) \rightarrow L_{\infty}(G)$

$$
S_{\varepsilon} f(\cdot)=\frac{1}{\varepsilon} \int_{0}^{\varepsilon} f(\cdot+t) d t, \quad f \in L_{\infty}(G),
$$

and define the function $\Phi_{\varepsilon}:=S_{\varepsilon} \chi_{(0, h)}$. Clearly, $\left\|\Phi_{\varepsilon}\right\|_{L_{1}(\mathbb{R})}=h,\left\|\Phi_{\varepsilon}^{\prime}\right\|_{L_{1}(\mathbb{R})}=2$, and

$$
\lim _{\varepsilon \rightarrow 0^{+}}\left\|D_{-}^{k} \Phi_{\varepsilon}\right\|_{L_{1}(\mathbb{R})}=\left\|D_{-}^{k} \chi_{(0, h)}\right\|_{L_{1}(\mathbb{R})}=\frac{2 h^{1-k}}{\Gamma(2-k)} .
$$

Plugging the latter relations into the first of inequality (31) we turn it into an equality. The proof is finished.

4.2 Case $G=\mathbb{R}_{+}, r=2$, and $k \in(0,1)$

For $k \in(0,1)$ and $h>0$, we define

$$
\tau_{h}(x):= \begin{cases}x^{1-k}-h^{-k} x, & x \in[0, h), \\ 0, & x \geq h .\end{cases}
$$

The following consequence of Theorem 5 holds true.

Corollary 7 Let $k \in(0,1)$, E be an ideal semi shift-invariant lattice on $\mathbb{R}_{+}$satisfying conditions (12) and (13), $E^{1}$ be the associated space to $E$. Then, for every $f \in L_{\infty, E}^{2}\left(\mathbb{R}_{+}\right)$and $h>0$, the sharp inequality

$$
\left\|D_{-}^{k} f\right\|_{L_{\infty}\left(\mathbb{R}_{+}\right)} \leq \frac{2 h^{-k}}{\Gamma(2-k)}\|f\|_{L_{\infty}\left(\mathbb{R}_{+}\right)}+\frac{\left\|\tau_{h}\right\|_{E^{1}}}{\Gamma(2-k)}\left\|f^{\prime \prime}\right\|_{E}
$$

holds true.

Proof For every $h>0$, we define the function

$$
\Omega(x):= \begin{cases}0, & x=0, \\ \frac{h^{-k}}{\Gamma(2-k)}, & x \in(0, h), \\ \frac{x^{-k}}{\Gamma(1-k)}, & x \geq h .\end{cases}
$$

Evidently, $\bigvee_{G} \Omega=\frac{2 h^{-k}}{\Gamma(2-k)}$ and $\mathcal{R}_{2-k}-\Omega^{[1]}=\frac{\tau_{h}}{\Gamma(2-k)} \in E^{1}$. Moreover, for every $f \in L_{\infty, E}^{2}\left(\mathbb{R}_{+}\right)$, we have (17). Indeed,

$$
\begin{aligned}
& D_{-}^{k} f(0)-\int_{0}^{+\infty} f(x) d \Omega(x) \\
& \quad=D_{-}^{k} f(0)-\frac{h^{-k} f(0)}{\Gamma(2-k)}+\frac{k h^{-k} f(h)}{\Gamma(2-k)}+\frac{k}{\Gamma(1-k)} \int_{h}^{\infty} \frac{f(x) d x}{x^{1+k}} \\
& \quad=\frac{k}{\Gamma(1-k)} \int_{0}^{h} \frac{f(0)-f(x)}{x^{1+k}} d x+\frac{k h^{-k} f(h)}{\Gamma(2-k)} \\
& \quad=-\frac{k}{\Gamma(1-k)} \int_{0}^{h} \int_{0}^{x} \frac{(x-t) f^{\prime \prime}(t)}{x^{1+k}} d t d x+\frac{k h^{-k}}{\Gamma(2-k)} \int_{0}^{h}(h-t) f^{\prime \prime}(t) d t \\
& \quad=\int_{0}^{+\infty}\left(\mathcal{R}_{2-k}(t)-\Omega^{[1]}(t)\right) f^{\prime \prime}(t) d t .
\end{aligned}
$$


Let us construct a family of functions $\left\{\Phi_{\varepsilon}\right\}_{\varepsilon>0} \subset W_{\infty, E}^{2}(G)$ satisfying conditions (19) and (28). For every $\varepsilon>0$, there exists a function $g_{\varepsilon} \in E,\left\|g_{\varepsilon}\right\|_{E} \leq 1$, such that

$$
\int_{0}^{h}\left(\mathcal{R}_{2-k}(x)-\Omega^{[1]}(x)\right) g_{\varepsilon}(x) d x>\left\|\mathcal{R}_{2-k}-\Omega^{[1]}\right\|_{E^{1}}-\varepsilon=\frac{\left\|\tau_{h}\right\|_{E^{1}}}{\Gamma(2-k)}-\varepsilon .
$$

Without loss of generality we may assume that $g_{\varepsilon}$ is non-negative on $G$ and $\operatorname{supp} g_{\varepsilon}=[0, h]$. Next, we define the function $\Phi_{\varepsilon}$ as the second integral of $g_{\varepsilon}$ :

$$
\Phi_{\varepsilon}(x):=\int_{0}^{h}(-x+t / 2) g_{\varepsilon}(t) d t+\int_{0}^{x}(x-t) g_{\varepsilon}(t) d t, \quad x \in \mathbb{R}_{+} .
$$

Clearly, $\Phi_{\varepsilon} \in W_{\infty, E}^{2}(G), \Phi_{\varepsilon}(x)=-\Phi_{\varepsilon}(0)=-\left\|\Phi_{\varepsilon}\right\|_{L_{\infty}\left(\mathbb{R}_{+}\right)}, x \geq h$. As a result,

$$
\begin{aligned}
\int_{0}^{+\infty} \Phi_{\varepsilon}(x) d \Omega(x) & =\frac{h^{-k}\left[\Phi_{\varepsilon}(0)-k \Phi_{\varepsilon}(h)\right]}{\Gamma(2-k)}-\frac{k(1-k)}{\Gamma(2-k)} \int_{h}^{+\infty} \frac{\Phi_{\varepsilon}(x)}{x^{1+k}} d x \\
& =\bigvee_{0}^{+\infty} \Omega \cdot\left\|\Phi_{\varepsilon}\right\|_{L_{\infty}\left(\mathbb{R}_{+}\right)}
\end{aligned}
$$

and

$$
\begin{aligned}
\int_{0}^{+\infty}\left(\mathcal{R}_{2-k}(x)-\Omega^{[1]}(x)\right) \Phi_{\varepsilon}^{\prime \prime}(x) d x & =\int_{0}^{h}\left(\mathcal{R}_{2-k}(x)-\Omega^{[1]}(x)\right) g_{\varepsilon}(x) d x \\
& >\left\|\mathcal{R}_{2-k}-\Omega^{[1]}\right\|_{E^{1}}-\varepsilon
\end{aligned}
$$

Therefore, the function $\Omega$ and the family of the functions $\left\{\Phi_{\varepsilon}\right\}_{\varepsilon>0}$ satisfy assumptions of Theorem 5 . Hence, inequality (32) holds true and is sharp.

Let us formulate the consequence of Corollaries 3, 4, and 7. For $s>1$, we set

$$
\varphi_{k, s}(x):=\int_{0}^{h}(-x+t / 2) \tau_{1}^{s^{\prime}-1}(t) d t+\int_{0}^{x}(x-t) \tau_{1}^{s^{\prime}-1}(t) d t, \quad x \in \mathbb{R}_{+},
$$

and $\Phi_{k, s}:=\left\|\varphi_{k, s}\right\|_{L_{s}\left(\mathbb{R}_{+}\right)}^{-1} \cdot \varphi_{k, s}$. Also, we define

$$
\Phi_{k, 1}(x)=\frac{1}{2} \cdot \max \left\{(1-k)^{1 / k}-2 x ;-(1-k)^{1 / k}\right\}, \quad x \in \mathbb{R}_{+} .
$$

Corollary 8 Let $k \in(0,1), 1 \leq s \leq \infty$, and $s^{\prime}=s /(s-1)$. Then, for every $f \in L_{\infty, s}^{2}\left(\mathbb{R}_{+}\right)$, the sharp inequality

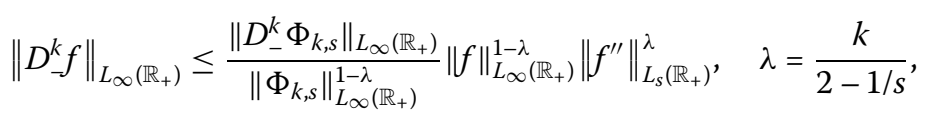

holds true.

4.3 Case $G=\mathbb{R}_{+}, 1<s \leq \infty$, and $k \in(1,2-1 / s)$

Let $1<s \leq \infty, s^{\prime}=s /(s-1)$, and $k \in(1,2-1 / s)$. Consider the set

$$
M:=\left\{(a, b) \in(0,1)^{2}: a \leq b\right\}
$$




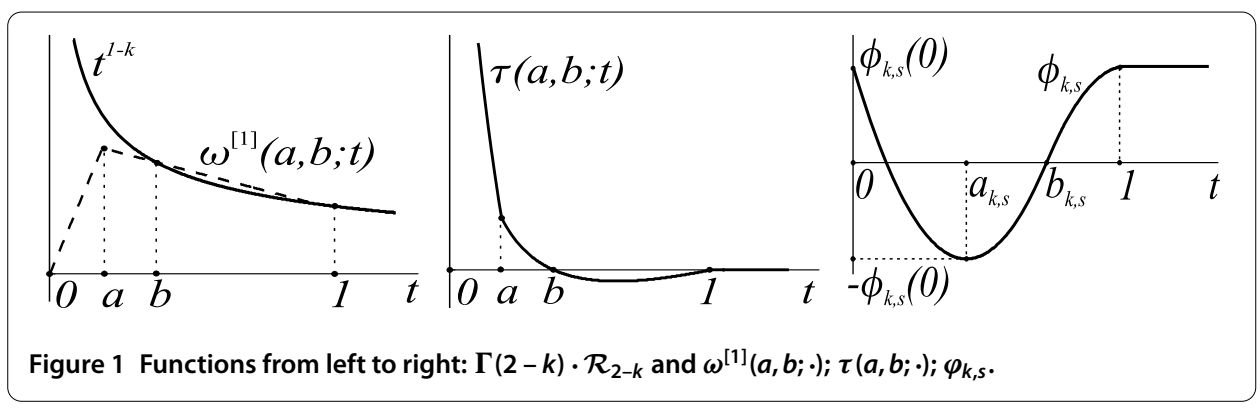

and, for every $(a, b) \in M$, we define the function

$$
\omega(a, b ; x)= \begin{cases}0, & x=0, \\ a^{-1}(1-b)^{-1} \cdot\left(1-b-\left(1-b^{1-k}\right)(1-a)\right), & x \in(0, a], \\ (1-b)^{-1} \cdot\left(1-b^{1-k}\right), & x \in(a, 1), \\ (1-k) x^{-k}, & x \geq 1 .\end{cases}
$$

For $x \in \mathbb{R}_{+}$, we consider the functions $\tau(a, b ; x):=\Gamma(2-k) \cdot \mathcal{R}_{2-k}(x)-\omega^{[1]}(a, b ; x)$ and

$$
\varphi(a, b ; x):=\int_{0}^{a}(-x+t / 2) \cdot \tau_{\left(s^{\prime}\right)}(a, b ; t) d t+\int_{0}^{x}(x-t) \cdot \tau_{\left(s^{\prime}\right)}(a, b ; t) d t,
$$

where $g_{\left(s^{\prime}\right)}:=|g|^{s^{\prime}-1} \operatorname{sign} g$. Below in Lemma 1 we shall show that system (33) has a unique solution $\left(a_{k, s}, b_{k, s}\right)$ on $M$. For convenience, we denote the functions $\omega\left(a_{k, s}, b_{k, s} ; \cdot\right)$, $\tau\left(a_{k, s}, b_{k, s} ;\right), \varphi\left(a_{k, s}, b_{k, s} ; \cdot\right)$ by $\omega_{k, s}, \tau_{k, s}, \varphi_{k, s}$, respectively. The graphs of the functions $\omega^{[1]}(a, b ; \cdot), \tau(a, b ; \cdot)$, and $\varphi_{k, s}$ are shown in Figure 1.

The next proposition is a consequence of Corollary 3.

Corollary 9 Let $1<s \leq \infty, s^{\prime}=s /(s-1), k \in(1,2-1 / s)$, and $\Phi_{k, s}:=\left\|\varphi_{k, s}\right\|_{L_{s}\left(\mathbb{R}_{+}\right)}^{-1} \cdot \varphi_{k, s}$. Then, for every $f \in L_{\infty, s}^{2}\left(\mathbb{R}_{+}\right)$, the sharp inequality

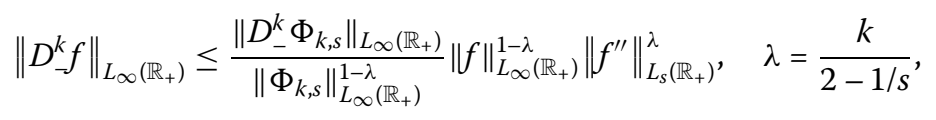

holds true.

We start with the proof of an auxiliary lemma.

Lemma 1 The system of the following equations has a unique solution on $M$ :

$$
\left\{\begin{array}{l}
F_{1}(a, b):=\int_{a}^{1} \tau_{\left(s^{\prime}\right)}(a, b ; t) d t=0, \\
F_{2}(a, b):=\int_{0}^{1} t \cdot \tau_{\left(s^{\prime}\right)}(a, b ; t) d t=0 .
\end{array}\right.
$$

We remark that in some cases the pair $\left(a_{k, s}, b_{k, s}\right)$ can be found explicitly, e.g. $a_{k, \infty}=\sqrt{2}-1$ and $b_{k, \infty}=1 / \sqrt{2}$.

Proof First, we observe that, for every $(a, b) \in M$, the function $\tau(a, b ; \cdot)$ is positive on $(0, b)$, is negative on $(b, 1)$, and $\operatorname{supp} \tau(a, b ; \cdot)=[0,1]$. Next, the functions $F_{1}$ and $F_{2}$ are continuous 
on $M$, and can be continuously extended on a wider set $\widetilde{M}:=\left\{(a, b) \in[0,1)^{2}: b>0, a \leq b\right\}$. Let us prove that system (33) has a unique solution on $\widetilde{M}$. First, we note that $F_{2}$ strictly increases in the variables $a$ and $b$, while $F_{1}$ strictly increases in $b$ and strictly decreases in $a$. Hence, system (33) could have only one solution. Next, we have

$$
\begin{aligned}
& \lim _{b \rightarrow 0^{+}} F_{2}(b, b) \leq \lim _{b \rightarrow 0^{+}}\left\{\int_{0}^{b} t^{(1-k)\left(s^{\prime}-1\right)+1} d t-\int_{b}^{1} t \cdot \tau_{\left(s^{\prime}\right)}(b, b ; t) d t\right\}=-\infty, \\
& \lim _{b \rightarrow 1^{-}} F_{2}(b, b)=\int_{0}^{1} t\left(t^{1-k}-t\right)^{s^{\prime}-1} d t>0, \\
& \lim _{b \rightarrow 1^{-}} F_{2}(0, b)=\int_{0}^{1} t\left(t^{1-k}-1+(1-k)(1-t)\right)^{s^{\prime}-1} d t>0 .
\end{aligned}
$$

Hence, there exist points $a^{*}, b^{*} \in(0,1)$ such that $F_{2}\left(0, b^{*}\right)=F_{2}\left(a^{*}, a^{*}\right)=0$. Taking into account continuity of the function $F_{2}$ and its monotonicity in both variables we conclude that, for every $a \in\left[0, a^{*}\right]$, there exists $b=\varrho(a) \in\left[a^{*}, b^{*}\right]$ such that $F_{2}(a, b)=0$. Moreover, the function $\varrho$ is continuous and is decreasing on the interval $\left[0, a^{*}\right]$ because it has an inverse function. Finally, we observe that

$$
\begin{aligned}
F_{1}\left(0, b^{*}\right) & =\frac{1}{b^{*}}\left\{b^{*} \int_{0}^{1} \tau_{\left(s^{\prime}\right)}\left(0, b^{*} ; t\right) d t\right\} \\
& >\frac{1}{b^{*}}\left\{\int_{0}^{b^{*}} t \cdot \tau_{\left(s^{\prime}\right)}\left(0, b^{*} ; t\right) d t\right\}=\frac{F_{2}\left(0, b^{*}\right)}{b^{*}}=0
\end{aligned}
$$

and

$$
F_{1}\left(a^{*}, a^{*}\right)=\int_{a^{*}}^{1} \tau_{\left(s^{\prime}\right)}\left(a^{*}, a^{*} ; t\right) d t<0 .
$$

Hence, there exists $a_{0} \in\left(0, a^{*}\right)$ such that $F_{1}\left(a_{0}, \varrho\left(a_{0}\right)\right)=0$. The latter implies that $\left(a_{0}, \varrho\left(a_{0}\right)\right) \in M$ and satisfies system (33).

Proof of Corollary 9 We set $\Omega:=\frac{\omega_{k, s}}{\Gamma(2-k)}$. It is easy to check that for $f \in L_{\infty, s}^{2}\left(\mathbb{R}_{+}\right)$,

$$
D_{-}^{k} f(0)-\int_{0}^{+\infty} f(t) d \Omega(t)=\int_{0}^{+\infty}\left(\mathcal{R}_{2-k}(t)-\Omega^{[1]}(t)\right) f^{\prime \prime}(t) d t .
$$

Moreover, $\Phi_{k, s} \in W_{\infty, s}^{2}\left(\mathbb{R}_{+}\right), \Phi_{k, s}(0)=-\Phi_{k, s}\left(a_{k, s}\right)=\left\|\Phi_{k, s}\right\|_{L_{\infty}\left(\mathcal{R}_{+}\right)}, \Phi_{k, s}(x)=\Phi_{k, s}(0)$, for every $x \geq h, \Phi_{k, s}$ decreases on $\left[0, a_{k, s}\right]$ and increases on $\left[a_{k, s},+\infty\right)$. Hence, the functions $\Omega$ and $\Phi_{k, s}$ satisfy the conditions of Corollary 3 .

\subsection{Case $G=\mathbb{R}, 1 \leq s \leq \infty$, and $k \in(0,1)$}

Let $k \in(0,1), 1 \leq s \leq \infty$, and $s^{\prime}=s /(s-1)$. For $p \in[0, k /(1-k)]$, we consider the function

$$
\omega(p ; x)= \begin{cases}0, & x \leq-p, \\ -(1+p)^{-1}, & x \in(-p, 1] \\ (1-k) x^{-k}, & x \geq 1 .\end{cases}
$$




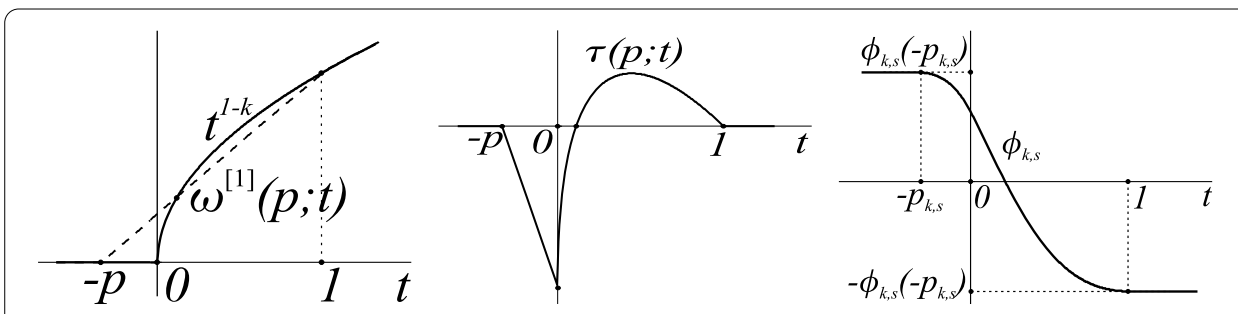

Figure 2 Functions from left to right: $\Gamma(2-k) \cdot \mathcal{R}_{2-k}$ and $\omega^{[1]}(p ; \cdot) ; \tau(p ; \cdot) ; \varphi_{k, s} \cdot$

For $x \in \mathbb{R}$, we consider the function $\tau(p ; x):=\Gamma(2-k) \mathcal{R}_{2-k}(x)-\omega^{[1]}(p ; x)$ and define

$$
\begin{aligned}
\varphi_{s}(p ; x): & =\frac{1}{2} \int_{-p}^{1} t \cdot \tau_{\left(s^{\prime}\right)}(p ; t) d t+\int_{-p}^{x}(x-t) \cdot \tau_{\left(s^{\prime}\right)}(p ; t) d t, \quad \text { when } s>1, \\
\varphi_{1}(p ; x): & : \begin{array}{ll}
(1+p) / 4, & x \leq-p, \\
(1+p-2 x) / 4, & x \in(-p, 1), \\
-(1+p) / 4, & x \geq 1 .
\end{array}
\end{aligned}
$$

Below in Lemma 2 we shall prove that the equations of (34) have a unique solution. For convenience, we denote by $p_{k, s}, s>1$, the solution to the first equation in (34) and by $p_{k, 1}$ the solution to the second equation in (34). In addition, we denote the functions $\omega\left(p_{k, s} ;\right), \tau\left(p_{k, s} ; \cdot\right)$, and $\varphi_{s}\left(p_{k, s} ; \cdot\right)$ by $\omega_{k, s}, \tau_{k, s}$, and $\varphi_{k, s}$, respectively. The graphs of the functions $\omega^{[1]}(p ; \cdot), \tau(p ; \cdot)$, and $\varphi_{k, s}$ are shown in Figure 2.

The following consequence of Corollaries 3 and 4 holds true.

Corollary 10 Let $1 \leq s \leq \infty, s^{\prime}=s /(s-1), k \in(0,1)$, and $h>0$. Then, for every $f \in L_{\infty, s}^{2}(\mathbb{R})$, the sharp inequality

$$
\left\|D_{-}^{k} f\right\|_{L_{\infty}(\mathbb{R})} \leq \frac{\left\|D_{-}^{k} \Phi_{k, s}\right\|_{L_{\infty}(\mathbb{R})}}{\left\|\Phi_{k, s}\right\|_{L_{\infty}(\mathbb{R})}^{1-\lambda}}\|f\|_{L_{\infty}(\mathbb{R})}^{1-\lambda}\left\|f^{\prime \prime}\right\|_{L_{s}(\mathbb{R})}^{\lambda}, \quad \lambda=\frac{k}{2-1 / s},
$$

where $\Phi_{k, s}:=\left\|\varphi_{k, s}^{\prime \prime}\right\|_{L_{s}(\mathbb{R})}^{-1} \cdot \varphi_{k, s}, s>1$, and $\Phi_{k, 1}:=\varphi_{k, 1}$, holds true.

We start with the proof of the following auxiliary lemma.

Lemma 2 Let $1<s \leq \infty, s^{\prime}=s /(s-1)$, and $k \in(0,1)$. Then the following equations have a unique solution on the interval $[0, k /(1-k)]$ :

$$
Z_{s}(p):=\int_{-p}^{1} \tau_{\left(s^{\prime}\right)}(p ; t) d t=0 \quad \text { and } \quad Z_{1}(p):=k^{k}(1-k)^{1-k}(1+p)-(2 k)^{1-k}=0 .
$$

We remark that for particular values of $s$ we can find $p_{k, s}$ explicitly, e.g. $p_{k, \infty}=1-2^{-k /(1-k)}$ and $p_{k, 2}=k /(2-k)$.

Proof The fact that the equation $Z_{1}(p)=0$ has a unique solution on the interval $[0, k /(1-$ $k)]$ is trivial. To prove that the equation $Z_{s}(p)=0$ also has a unique solution on the same interval we observe that $Z_{s}$ is continuous and strictly decreases on the interval $[0, k /(k-1)]$, 


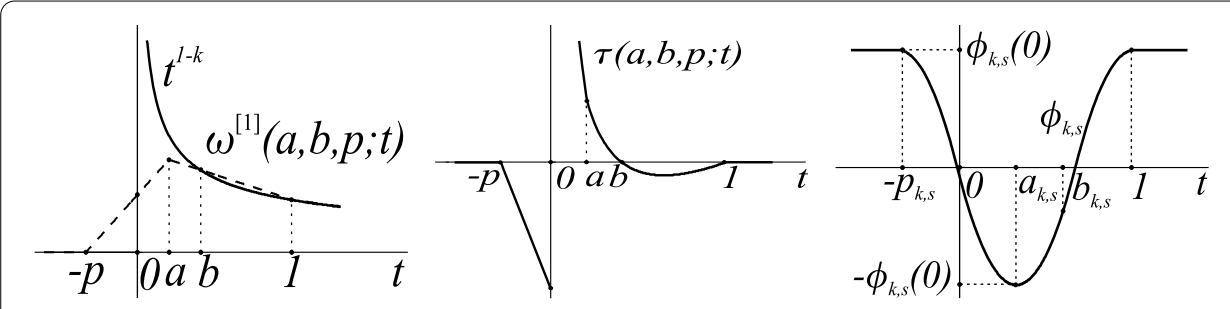

Figure 3 Functions from left to right: $\Gamma(2-k) \cdot \mathcal{R}_{2-k}$ and $\omega^{[1]}(a, b, p ; \cdot) ; \tau(a, b, p ; \cdot) ; \varphi_{k, s}$.

and attains values of opposite signs at points 0 and $k /(k-1)$. Thus the equation $Z_{s}(p)=0$ has a unique solution on $[0, k /(1-k)]$.

Proof of Corollary 10 We set $\Omega:=\frac{\omega_{k, s}}{\Gamma(2-k)}$. Similarly to Section 4.2 we can check that, for every $f \in L_{\infty, s}^{2}(\mathbb{R})$, equality (17) holds true. Moreover, if $s>1$, we see that $\Phi_{k, s}$ decreases on $\mathbb{R}, \Phi_{k, s} \in W_{\infty, s}^{2}(\mathbb{R}), \Phi_{k, s}\left(-p_{k, s}\right)=-\Phi_{k, s}(1)=\left\|\Phi_{k, s}\right\|_{L_{\infty}(\mathbb{R})}$. Hence, the functions $\Omega$ and $\Phi_{k, s}$ satisfy the conditions of Corollary 3 . In turn, for $s=1$ we can check that the functions $\Omega$ and $\Phi_{k, 1}$ satisfy the conditions of Corollary 4 .

4.5 Case $G=\mathbb{R}, 1<s \leq \infty$, and $k \in(1,2-1 / s)$

Let $1<s \leq \infty, s^{\prime}=s /(s-1)$, and $k \in(1,2-1 / s)$. Consider the set $S:=M \times[0,+\infty)$, where the set $M$ was defined in Section 4.3, and, for every $(a, b, p) \in S$, we define

$$
\omega(a, b, p ; x)= \begin{cases}0, & x \leq-p, \\ \frac{1-b+\left(1-b^{1-k}\right)(a-1)}{(1-b)(a+p)}, & x \in[-p, a], \\ (1-b)^{-1} \cdot\left(b^{1-k}-1\right), & x \in[a, 1], \\ (1-k) x^{-k}, & x \geq 1 .\end{cases}
$$

For $x \in \mathbb{R}$, we consider functions $\tau(a, b, p ; x):=\Gamma(2-k) \cdot \mathcal{R}_{2-k}(x)-\omega^{[1]}(a, b, p ; x)$ and

$$
\varphi(a, b, p ; x):=\frac{1}{2} \int_{-p}^{a} t \cdot \tau_{\left(s^{\prime}\right)}(a, b, p ; t) d t+\int_{-p}^{x}(x-t) \cdot \tau_{\left(s^{\prime}\right)}(a, b, p ; t) d t .
$$

Below in Lemma 3 we shall prove that the system of equations (35) has at least one solution on $S$. Let $\left(a_{k, s}, b_{k, s}, p_{k, s}\right)$ be one of such solutions and, for simplicity of notation, we denote the functions $\omega\left(a_{k, s}, b_{k, s}, p_{k, s} \cdot\right), \tau\left(a_{k, s}, b_{k, s}, p_{k, s} ; \cdot\right)$, and $\varphi\left(a_{k, s}, b_{k, s}, p_{k, s} ; \cdot\right)$ by $\omega_{k, s}, \tau_{k, s}$ and $\varphi_{k, s}$, respectively. The graphs of the functions $\omega^{[1]}(a, b, p ; \cdot), \tau(a, b, p ; \cdot)$, and $\varphi_{k, s}$ are shown in Figure 3.

Next, we set $\Phi_{k, s}:=\left\|\varphi_{k, s}^{\prime \prime}\right\|_{L_{s}(\mathbb{R})}^{-1} \cdot \varphi_{k, s}$. The following consequence of Corollary 3 holds true.

Corollary 11 Let $1<s \leq \infty, s^{\prime}=s /(s-1), k \in(1,2-1 / s)$, and $h>0$. Then, for every function $f \in L_{\infty, s}^{2}(\mathbb{R})$, the sharp inequalities

$$
\left\|D_{-}^{k} f\right\|_{L_{\infty}(\mathbb{R})} \leq \frac{\left\|D_{-}^{k} \Phi_{k, s}\right\|_{L_{\infty}(\mathbb{R})}}{\left\|\Phi_{k, s}\right\|_{L_{\infty}(\mathbb{R})}^{1-\lambda}}\|f\|_{L_{\infty}(\mathbb{R})}^{1-\lambda}\left\|f^{\prime \prime}\right\|_{L_{s}(\mathbb{R})}^{\lambda}, \quad \lambda=\frac{k}{2-1 / s},
$$

hold true.

We start with the proof of the following auxiliary lemma. 
Lemma 3 The following system of equations has a solution on S:

$$
\left\{\begin{array}{l}
Z_{1}(a, b, p):=\int_{-p}^{a} \tau_{\left(s^{\prime}\right)}(a, b, p ; t) d t=0, \\
Z_{2}(a, b, p):=\int_{a}^{1} \tau_{\left(s^{\prime}\right)}(a, b, p ; t) d t=0, \\
Z_{3}(a, b, p):=\int_{-p}^{1} t \cdot \tau_{\left(s^{\prime}\right)}(a, b, p ; t) d t=0 .
\end{array}\right.
$$

For particular values of $s$ the triple $\left(a_{k, s}, b_{k, s}, p_{k, s}\right)$ can be found explicitly, e.g. $p_{k, \infty}=a_{k, \infty}=$ $1 / 3$ and $b_{k, \infty}=2 / 3$.

Proof First, we observe that the function $Z_{2}$ is continuous on $S$, strictly decreases in the variable $a$, strictly increases in the variable $b$, and is constant in the variable $p$. In addition, for every $a \in(0,1), Z_{2}(a, a, 0)<0$ and $\lim _{b \rightarrow 1-0} Z_{2}(a, b, 0)>0$. The latter and monotonicity of $Z_{2}$ in the variable $b$ imply that there exists a strictly increasing function $\gamma:(0,1) \rightarrow$ $\mathbb{R}$ such that $Z_{2}(a, \gamma(a), 0)=0$. Moreover, continuity of the function $\gamma$ follows from its monotonicity and continuity of the function $Z_{2}$.

Next, we consider the function $Z_{1}$. Evidently, $Z_{1}$ is continuous on $S$, strictly increases in the variables $a$ and $b$, and strictly decreases in the variable $p$. Since, for every $a \in(0,1)$, $Z_{1}(a, \gamma(a), 0)>0$, and $\lim _{p \rightarrow-\infty} Z_{1}(a, \gamma(a), p)=-\infty$, we conclude that there exists a function $\delta:(0,1) \rightarrow \mathbb{R}$ such that, for every $a \in(0,1), Z_{1}(a, \gamma(a), \delta(a))=0$. Since $Z_{1}$ is continuous on $S$ and $\delta$ is monotone, we conclude that $\delta$ is also continuous on $(0,1)$. Therefore, for every $a \in(0,1)$, we have $Z_{1}(a, \gamma(a), \delta(a))=Z_{2}(a, \gamma(a), \delta(a))=0$. Now, we set $b^{*}=\lim _{a \rightarrow+0} \gamma(a)$.

Since

$$
\begin{aligned}
\lim _{a \rightarrow 0^{+}} Z_{2}\left(a, \frac{1}{2}, 0\right)= & \int_{0}^{1 / 2}\left(t^{1-k}-1+2\left(1-2^{k-1}\right)(1-t)\right)^{s^{\prime}-1} d t \\
& -\int_{1 / 2}^{1}\left(t^{1-k}-1+2\left(1-2^{k-1}\right)(1-t)\right)^{s^{\prime}-1} d t>0
\end{aligned}
$$

and

$$
\lim _{a \rightarrow 0^{+}} Z_{2}\left(a, \frac{1}{2}, 0\right)=\int_{0}^{1 / 2}\left(t^{1-k}-t\right)^{s^{\prime}-1} d t-\int_{1 / 2}^{1}\left(t^{1-k}-t\right)^{s^{\prime}-1} d t>0,
$$

we conclude that $b^{*} \in(0,1)$.

Finally, note that $Z_{3}$ is also continuous on the $S$ function, and

$$
\begin{aligned}
\lim _{a \rightarrow 0^{+}} Z_{3}(a, \gamma(a), \delta(a))= & Z_{3}\left(+0, b^{*}, 0\right)=\int_{0}^{b^{*}} t \cdot \tau_{k}^{s^{\prime}-1}\left(+0, b^{*}, 0\right) d t \\
& -\int_{b^{*}}^{1} t \cdot \tau_{k}^{s^{\prime}-1}\left(+0, b^{*}, 0\right) d u \\
& <b^{*}\left[\int_{0}^{b^{*}} \tau_{k}^{s^{\prime}-1}(a, b, p ; t) d t-\int_{b^{*}}^{1} \tau_{k}^{s^{\prime}-1}(a, b, p ; t) d t\right] \\
= & b^{*} Z_{2}\left(+0, b^{*}, 0\right)=0, \\
\lim _{a \rightarrow 1^{-}} Z_{3}(a, \gamma(a), \delta(a))> & \int_{0}^{1} t\left(t^{1-k}-1\right)^{s^{\prime}-1} d t>0 .
\end{aligned}
$$

Hence, there exists $a \in(0,1)$ such that $Z_{3}(a, \gamma(a), \delta(a))=0$. 
Proof of Corollary 11 We set $\Omega:=\frac{\omega_{k, s}}{\Gamma(2-k)}$. It is easy to check that (17) holds true, for every $f \in L_{\infty, s}^{2}\left(\mathbb{R}_{+}\right)$. Moreover, $\Phi_{k, s} \in W_{\infty, s}^{2}(\mathbb{R}), \Phi_{k, s}\left(-p_{k, s}\right)=-\Phi_{k, s}\left(a_{k, s}\right)=\left\|\Phi_{k, s}\right\|_{L_{\infty}(\mathbb{R})}, \Phi_{k, s}(x)=$ $\left\|\Phi_{k, s}\right\|_{L_{\infty}(\mathbb{R})}$, for every $x \geq 1, \Phi_{k, s}$ is non-increasing on $\left(-\infty, a_{k, s}\right]$ and non-decreasing on $\left[a_{k, s},+\infty\right)$. Hence, the functions $\Omega$ and $\Phi_{k, s}$ satisfy the conditions of Corollary 3 , which finishes the proof.

\section{Applications}

In this section we consider applications of results of this paper. We devote Section 5.1 to consequences related to the Kolmogorov problem for three numbers and in Section 5.2 we obtain sharp Kolmogorov type inequalities for the weighted norms of fractional powers of the operator $\mathscr{D}=x \frac{d}{d x}$ obtained by the Hadamard fractional derivative.

\subsection{The Kolmogorov problem for three numbers}

Let $G=\mathbb{R}$ or $G=\mathbb{R}_{+}, 1 \leq p, q, s \leq \infty, r \in \mathbb{N}$, and $k \in(0, r) \backslash \mathbb{N}$. The Kolmogorov problem for three numbers (see [3]) consists in finding necessary and sufficient conditions on three positive numbers $M_{0}, M_{k}$, and $M_{r}$ that guarantee the existence of a function $f \in L_{p, s}^{r}(G)$ satisfying the equalities

$$
\|f\|_{L_{p}(G)}=M_{0}, \quad\left\|D_{-}^{k} f\right\|_{L_{q}(G)}=M_{k}, \quad\left\|f^{(r)}\right\|_{L_{s}(G)}=M_{r} .
$$

For an overview of known results in this direction, we refer the reader to [3, 4] and references therein. Using similar arguments and combining them with results of the previous section we deduce the following consequences.

Theorem 7 Let $G=\mathbb{R}$ or $G=\mathbb{R}_{+}, 1<s \leq \infty, p=q=\infty, k \in(0,2-1 / s), r=2$, and $M_{0}$, $M_{k}, M_{2}$ be positive numbers. Assume that $K$ is a sharp constant in inequality (7) and there exists a non-negative function turning (7) into an equality. Then there exists a function $f \in L_{\infty, s}^{2}(G)$ satisfying equalities (36) if and only if $M_{k} \leq K \cdot M_{0}^{1-\lambda} M_{2}^{\lambda}$ where $\lambda=\frac{k}{2-1 / s}$.

Theorem 8 Let $G=\mathbb{R}$ or $G=\mathbb{R}_{+}, s=1, p=q=\infty, k \in(0,2-1 / s), r=2$, and $M_{0}, M_{k}$, $M_{2}$ be positive numbers. Assume that $K$ is a sharp constant in inequality (7). Then there exists a function $f \in L_{\infty, s}^{r}(G)$ satisfying equalities (36) if and only if $M_{k}<K \cdot M_{0}^{1-\lambda} M_{2}^{\lambda}$ where $\lambda=\frac{k}{2-1 / s}$.

\subsection{Sharp Kolmogorov type inequalities for the Hadamard fractional derivatives}

Let $\mathscr{D}$ be the operator mapping every differentiable function $f: \mathbb{R}_{+} \rightarrow \mathbb{R}$ into the function $\mathscr{D} f(x)=x f^{\prime}(x), x \in \mathbb{R}_{+}$, i.e. $\mathscr{D}=x \frac{d}{d x}$. The fractional power, $k \in \mathbb{R}_{+} \backslash \mathbb{N}$, of the operator $\mathscr{D}$ is obtained by the Hadamard fractional differentiation operator $\mathscr{D}_{ \pm}^{k}$ (see [7, Section 18]) which is defined as follows: for $f: \mathbb{R}_{+} \rightarrow \mathbb{R}$ and $x \in \mathbb{R}_{+}$,

$$
\begin{aligned}
& \mathscr{D}_{+}^{k} f(x)=\frac{1}{\varkappa(k, r)} \int_{0}^{1} \sum_{m=0}^{r}(-1)^{m}\left(\begin{array}{c}
r \\
m
\end{array}\right) f\left(u^{m} x\right) \frac{d u}{u|\ln u|^{1+k}}, \\
& \mathscr{D}_{-}^{k} f(x)=\frac{1}{\varkappa(k, r)} \int_{1}^{+\infty} \sum_{m=0}^{r}(-1)^{m}\left(\begin{array}{c}
r \\
m
\end{array}\right) f\left(u^{m} x\right) \frac{d u}{u|\ln u|^{1+k}},
\end{aligned}
$$

where $r \in \mathbb{N}, r>k$, and $\varkappa(k, r)$ was defined in (6). Some Kolmogorov type inequalities for the Hadamard fractional derivatives were considered in [40] 
For an arbitrary function $f: \mathbb{R}_{+} \rightarrow \mathbb{R}$, let us define the function $g: \mathbb{R} \rightarrow \mathbb{R}$ as follows: $g(t)=f\left(e^{t}\right), t \in \mathbb{R}$. Then, for every $x \in \mathbb{R}_{+}$, we have $\mathscr{D}_{ \pm}^{k} f(x)=D_{ \pm}^{k} g(\ln x)$. As a result, $\left\|\mathscr{D}_{ \pm}^{k} f\right\|_{L_{\infty}\left(\mathbb{R}_{+}\right)}=\left\|D_{ \pm}^{k} g\right\|_{L_{\infty}(\mathbb{R})}$ and, for every $1 \leq s<\infty$,

$$
\int_{0}^{+\infty}\left|\mathscr{D}_{ \pm}^{k} f(x)\right|^{s} \frac{d x}{x}=\left\|D_{ \pm}^{k} g\right\|_{L_{s}(\mathbb{R})}^{s} .
$$

The latter formula allows deducing sharp Kolmogorov type inequalities for the weighted $L_{s}$-norms of the Hadamard fractional derivatives from sharp Kolmogorov type inequalities for $L_{s}$-norms of the Marchaud fractional derivatives. Let us present rigorous statements. For $1 \leq s \leq \infty$, by $\mathscr{L}_{s}$ we denote the space of the functions $f: \mathbb{R}_{+} \rightarrow \mathbb{R}$ endowed with the norm

$$
\|f\|_{\mathscr{L}_{s}}= \begin{cases}\left(\int_{0}^{+\infty}|f(x)|^{s} \frac{d x}{x}\right)^{1 / s}, & 1 \leq s<\infty \\ \|f\|_{L_{\infty}\left(\mathbb{R}_{+}\right)}, & s=\infty\end{cases}
$$

For $r \in \mathbb{N}$, let $\mathscr{L}_{\infty, s}^{r}$ be the space of the functions $f \in L_{\infty}\left(\mathbb{R}_{+}\right)$such that $f^{(r-1)}$ is locally absolutely continuous on $\mathbb{R}_{+}$, and $f^{(r)} \in \mathscr{L}_{s}$. From the above arguments we conclude that the following proposition holds true.

Theorem 9 Let the Kolmogorov type inequality (1) with sharp constant $K$ hold true for some collection of parameters $1 \leq p, q, s \leq \infty, r \in \mathbb{N}, k \in(0, r)$, and $\mu, \lambda \in \mathbb{R}, 0 \leq \mu=1-\lambda \leq$ 1. Then for the same collection of parameters and, for every $f \in \mathscr{L}_{p, s}^{r}$, the sharp inequality

$$
\left\|\mathscr{D}_{ \pm}^{k} f\right\|_{\mathscr{L}_{q}} \leq K\|f\|_{\mathscr{L}_{p}}^{\mu}\left\|\mathscr{D}^{r} f\right\|_{\mathscr{L}_{s}}^{\lambda}
$$

holds true.

Combining Theorem 9 with Corollaries 10 and 11 we obtain the following.

Corollary 12 Let $1 \leq s \leq \infty, s^{\prime}=s /(s-1), k \in(0,2-1 / s)$, and $\lambda=k /(2-1 / s)$. Then, for every function $f \in \mathscr{L}_{\infty, s}^{2}$, the sharp inequality

$$
\left\|\mathscr{D}_{ \pm}^{k} f\right\|_{\mathscr{L}_{\infty}} \leq \frac{\left\|D_{-}^{k} \Phi_{k, s}\right\|_{L_{\infty}(\mathbb{R})}}{\left\|\Phi_{k, s}\right\|_{L_{\infty}(\mathbb{R})}^{1-\lambda}}\|f\|_{\mathscr{L}_{\infty}}^{1-\lambda}\left\|\mathscr{D}^{2} f\right\|_{\mathscr{L}_{s}}^{\lambda}
$$

holds true, where the function $\Phi_{k, s}$ was defined in Sections 4.2-4.5.

\section{Competing interests}

The authors declare that they have no competing interests.

\section{Authors' contributions}

All authors made an equal contribution to the paper, and they have read and approved the final manuscript.

Received: 11 August 2014 Accepted: 2 December 2014 Published: 17 Dec 2014

References

1. Arestov, W, Gabushin, VN: Best approximation of unbounded operators by bounded operators. Russ. Math. (Izv. VUZ) 39(11), 38-63 (1995)

2. Arestov, W: Approximation of unbounded operators by bounded operators and related extremal problems. Russ. Math. Surv. 51(6), 1093-1126 (1996) 
3. Kolmogorov, AN: On inequalities between the upper bounds of the successive derivatives of an arbitrary function on an infinite interval. In: Selected Works. Mathematics and Mechanics, pp. 252-263. Nauka, Moscow (1985)

4. Babenko, VF, Korneichuk, NP, Kofanov, VA, Pichugov, SA: Inequalities for Derivatives and Their Applications. Naukova Dumka, Kiev (2003)

5. Kwong, MK, Zettl, A: Norm Inequalities for Derivatives and Differences. Lecture Notes in Mathematics, vol. 1536. Springer, Berlin (1992)

6. Mitrinovic, DS, Pecaric, J, Fink, AM: Inequalities Involving Functions and Their Integrals and Derivatives. Kluwer Academic, Dordrecht (1991)

7. Samko, SG, Kilbas, AA, Marichev, Ol: Fractional Integrals and Derivatives: Theory and Applications. Taylor \& Francis, London (2002)

8. Geissberg, SP: Generalization of Hadamard's inequality. Sb. Nauch. Tr. Leningr. Mekh. Inst. 50, 42-54 (1965)

9. Geissberg, SP: Fractional derivatives of functions bounded on the axis. Izv. Vyš̌. Učebn. Zaved., Mat. 11, 51-69 (1968)

10. Arestov, W: Inequalities for fractional derivatives on the half-line. In: Approximation Theory, vol. 4, pp. 19-34. Banach Center Publication, Warsaw (1979)

11. Magaril-Il'jaev, GG, Tihomirov, VM: On the Kolmogorov inequality for fractional derivatives on the half-line. Anal. Math. 7(1), 37-47 (1981)

12. Babenko, VF, Churilova, MS: On inequalities of Kolmogorov type for derivatives of fractional order. Bull. Dnepropetrovsk Univ. Math. 6, 16-20 (2001)

13. Babenko, VF, Churilova, MS: Kolmogorov type inequalities for hypersingular integrals with homogeneous characteristic. Banach J. Math. Anal. 1(1), 66-77 (2007)

14. Babenko, VF, Pichugov, SA: Sharp estimates of the norms of fractional derivatives of functions of several variables satisfying Hölder conditions. Math. Notes 87(1-2), 22-30 (2010)

15. Babenko, VF, Parfinovych, NV, Pichugov, SA: Kolmogorov-type inequalities for norms of Riesz derivatives of functions of several variables with Laplacian bounded in $L_{\infty}$ and related problems. Math. Notes 95(1), 3-14 (2014)

16. Motornyi, VP, Babenko, VF, Dovgoshei, AA, Kuznetsova, Ol: Approximation Theory and Harmonic Analysis. Naukova Dumka, Kiev (2012)

17. Gabushin, VN: Inequalities for the norms of a function and its derivatives in metric $L_{p}$. Math. Notes 1(3), 194-198 (1967)

18. Krein, SG, Petunin, Jl, Semenov, EM: Interpolation of Linear Operators. Am. Math. Soc., Groningen (1982)

19. Marchaud, A: Sur les derivées et sur les différences des fonctions de variables réelles. J. Math. Pures Appl. 6, 337-425 (1927)

20. Babenko, VF, Parfinovych, NV: Kolmogorov type inequalities for norms of Riesz derivatives of multivariate functions and some applications. Proc. Steklov Inst. Math. 277(1), suppl., 9-20 (2012)

21. Buslaev, AP, Tikhomirov, VM: Inequalities for derivatives in the multidimensional case. Math. Notes 25(1), 32-40 (1979)

22. Babenko, VF, Churilova, MS: On Kolmogorov type inequalities for fractional derivatives of the functions defined on the real line. Bull. Dnepropetrovsk Univ. Math. 13, 28-34 (2008)

23. Stechkin, SB: Best approximation of linear operators. Math. Notes 1(2), 91-99 (1967)

24. Gabushin, VN: Best approximations of functionals on certain sets. Math. Notes 8(5), $780-785$ (1970)

25. Kilbas, AA, Srivastava, HM, Trujillo, Jj: Theory and Applications of Fractional Differential Equations. Elsevier, Amsterdam (2006)

26. Krasnosel'skii, MA, Rutitskii, YB: Convex Functions and Orlich Spaces. Noordhoff, Groningen (1961)

27. Chui, CK: An Introduction to Wavelets. Academic Press, London (1992)

28. Arestov, W: On exact inequalities between norms of functions and its derivatives. Acta Sci. Math. 33(3-4), 243-267 (1972) (in Russian)

29. Gabushin, VN: On the best approximation of the differentiation operator on the half-line. Math. Notes 6(5), 804-810 (1969)

30. Taikov, LV: Kolmogorov-type inequalities and the best formulas for numerical differentiation. Math. Notes 4(2), 631-634 (1968)

31. Kolmogorov, AN: On inequalities between the upper bounds of the successive derivatives of an arbitrary function on the infinite interval. Uch. Zap. MGU, Mat. 30, 3-13 (1939)

32. Domar, Y: An extremal problem related to Kolmogoroff's inequality for bounded functions. Ark. Mat. 7(5), 433-441 (1968)

33. Arestov, W: On the best approximation of differentiation operators. Math. Notes 1(2), 100-103 (1967)

34. Buslaev, AP: Approximation of a differentiation operator. Math. Notes 29(5), 372-378 (1981)

35. Stein, EM: Functions of exponential type. Ann. Math. 65(3), 582-592 (1957)

36. Babenko, VF, Pichugov, SA: About one method of Stein. In: Approximation of Functions and Summation of Series, vol. 43, pp. 420-422. Dnepropetrovsk State University, Dnepropetrovsk (1991)

37. Korneichuck, NP, Babenko, VF, Ligun, AA: Extremal Properties of Polynomials and Splines. Nova Science Publishers, New York (1996)

38. Arestov, W: On the best uniform approximation of differentiation operators. Math. Notes 5(3), 167-173 (1969)

39. Buslaev, AP, Magaril-II'yaev, GG, Tikhomirov, VM: Existence of extremal functions in inequalities for derivatives. Math. Notes 32(6), 898-904 (1982)

40. Babenko, VF, Parfinovych, NV: On Kolmogorov type inequalities for the Hadamard fractional derivatives of functions defined on a semi-axis. Bull. Dnepropetrovsk Univ. Math. 17(6), 31-35 (2009) (in Russian)

\subsection{6/1029-242X-2014-504}

Cite this article as: Babenko et al.: Kolmogorov type inequalities for the Marchaud fractional derivatives on the real line and the half-line. Journal of Inequalities and Applications 2014, 2014:504 